Proceedings of the Edinburgh Mathematical Society (2005) 48, 705-742 (C)

DOI:10.1017/S0013091503000695 Printed in the United Kingdom

\title{
DETERMINANT EXPRESSIONS FOR HYPERELLIPTIC FUNCTIONS
}

\section{YOSHIHIRO ÔNISHI}

Faculty of Humanities and Social Sciences, Iwate University,

Morioka 020-8550, Japan (onishi@iwate-u.ac.jp)

(Received 19 August 2003)

\begin{abstract}
In this paper we give an elegant generalization of the formula of Frobenius-Stickelberger from elliptic curve theory to all hyperelliptic curves. A formula of Kiepert type is also obtained by a limiting process from this generalization. In the appendix a determinant expression of D. G. Cantor is also derived.
\end{abstract}

Keywords: Abelian functions; Frobenius-Stickelberger-type formula; Kiepert-type formula

2000 Mathematics subject classification: Primary 11G30; 11G10; 14H45

\section{Introduction}

Let $\sigma(u)$ and $\wp(u)$ be the usual functions from the classical theory of elliptic functions. The following two formulae were found in the nineteenth century. The first one is

$$
\begin{gathered}
(-1)^{(n-1)(n-2) / 2} 1 ! 2 ! \cdots(n-1) ! \frac{\sigma\left(u^{(1)}+u^{(2)}+\cdots+u^{(n)}\right) \prod_{i<j} \sigma\left(u^{(i)}-u^{(j)}\right)}{\sigma\left(u^{(1)}\right)^{n} \sigma\left(u^{(2)}\right)^{n} \cdots \sigma\left(u^{(n)}\right)^{n}} \\
=\left|\begin{array}{cccccc}
1 & \wp\left(u^{(1)}\right) & \wp^{\prime}\left(u^{(1)}\right) & \wp^{\prime \prime}\left(u^{(1)}\right) & \cdots & \wp^{(n-2)}\left(u^{(1)}\right) \\
1 & \wp\left(u^{(2)}\right) & \wp^{\prime}\left(u^{(2)}\right) & \wp^{\prime \prime}\left(u^{(2)}\right) & \cdots & \wp^{(n-2)}\left(u^{(2)}\right) \\
\vdots & \vdots & \vdots & \vdots & \ddots & \vdots \\
1 & \wp\left(u^{(n)}\right) & \wp^{\prime}\left(u^{(n)}\right) & \wp^{\prime \prime}\left(u^{(n)}\right) & \cdots & \wp^{(n-2)}\left(u^{(n)}\right)
\end{array}\right| .
\end{gathered}
$$

This formula appeared in the paper of Frobenius and Stickelberger [11]. The second one is

$$
(-1)^{n-1}(1 ! 2 ! \cdots(n-1) !)^{2} \frac{\sigma(n u)}{\sigma(u)^{n^{2}}}=\left|\begin{array}{cccc}
\wp^{\prime} & \wp^{\prime \prime} & \cdots & \wp^{(n-1)} \\
\wp^{\prime \prime} & \wp^{\prime \prime \prime} & \cdots & \wp^{(n)} \\
\vdots & \vdots & \ddots & \vdots \\
\wp^{(n-1)} & \wp^{(n)} & \cdots & \wp^{(2 n-3)}
\end{array}\right|(u) .
$$

Although this formula can be obtained by a limiting process from (1.1), it was found before (1.1) by Kiepert [14].

If we set $y(u)=\frac{1}{2} \wp^{\prime}(u)$ and $x(u)=\wp(u)$, then we have the equation $y(u)^{2}=x(u)^{3}+\cdots$, which is a defining equation of the elliptic curve to which the functions $\wp(u)$ and $\sigma(u)$ 
are attached. Here the complex number $u$ and the coordinate $(x(u), y(u))$ correspond by the identity

$$
u=\int_{\infty}^{(x(u), y(u))} \frac{\mathrm{d} x}{2 y}
$$

with an appropriate choice of path of the integral. Then (1.1) and (1.2) are easily rewritten as

$$
\begin{aligned}
& \frac{\sigma\left(u^{(1)}+u^{(2)}+\cdots+u^{(n)}\right) \prod_{i<j} \sigma\left(u^{(i)}-u^{(j)}\right)}{\sigma\left(u^{(1)}\right)^{n} \sigma\left(u^{(2)}\right)^{n} \cdots \sigma\left(u^{(n)}\right)^{n}} \\
& =\left|\begin{array}{ccccccc}
1 & x\left(u^{(1)}\right) & y\left(u^{(1)}\right) & x^{2}\left(u^{(1)}\right) & y x\left(u^{(1)}\right) & x^{3}\left(u^{(1)}\right) & \cdots \\
1 & x\left(u^{(2)}\right) & y\left(u^{(2)}\right) & x^{2}\left(u^{(2)}\right) & y x\left(u^{(2)}\right) & x^{3}\left(u^{(2)}\right) & \cdots \\
\vdots & \vdots & \vdots & \vdots & \vdots & \vdots & \ddots \\
1 & x\left(u^{(n)}\right) & y\left(u^{(n)}\right) & x^{2}\left(u^{(n)}\right) & y x\left(u^{(n)}\right) & x^{3}\left(u^{(n)}\right) & \cdots
\end{array}\right|
\end{aligned}
$$

and

$$
1 ! 2 ! \cdots(n-1) ! \frac{\sigma(n u)}{\sigma(u)^{n^{2}}}=\left|\begin{array}{cccccc}
x^{\prime} & y^{\prime} & \left(x^{2}\right)^{\prime} & (y x)^{\prime} & \left(x^{3}\right)^{\prime} & \cdots \\
x^{\prime \prime} & y^{\prime \prime} & \left(x^{2}\right)^{\prime \prime} & (y x)^{\prime \prime} & \left(x^{3}\right)^{\prime \prime} & \cdots \\
\vdots & \vdots & \vdots & \vdots & \vdots & \ddots \\
x^{(n-1)} & y^{(n-1)} & \left(x^{2}\right)^{(n-1)} & (y x)^{(n-1)} & \left(x^{3}\right)^{(n-1)} & \ldots
\end{array}\right|(u),
$$

respectively.

The author recently gave a generalization of the formulae (1.3) and (1.4) to the case of genus two in $[\mathbf{1 9}]$ and to the case of genus three in $[\mathbf{2 0}]$. The aim of this paper is to generalize $(1.3),(1.4)$ and the results in $[\mathbf{1 9}, \mathbf{2 0}]$ to all hyperelliptic curves (see Theorems 8.2 and 9.3). The idea of our generalization arises from the highly original paper [12] of Grant. It can be summarized in a phrase, 'Think not on the Jacobian but on the curve itself'.

Fay's famous formula (44) [10, p. 33], which generalizes an addition formula on the Jacobian variety, known as Schottky-Klein, is another generalization of (1.3). The author does not know whether Fay's formula is able to yield a generalization of (1.4). Our formula is quite elegant in comparison with Fay's one and naturally gives a generalization of (1.4). Though no explicit connection between Fay's formula and ours is known at present, a paper that seems to investigate this problem recently appeared [9].

We now present the minimal fundamentals needed to explain our results. Let $f(x)$ be a monic polynomial in $x$ of degree $2 g+1$ with $g$ a positive integer. Assume that $f(x)=0$ has no multiple roots. Let $C$ be the hyperelliptic curve defined by $y^{2}=f(x)$. Then $C$ is of genus $g$ and is ramified at infinity. We denote by $\infty$ the unique point at infinity on $C$. Let $\boldsymbol{C}^{g}$ be the coordinate space of all vectors of integrals

$$
\left(\int_{\infty}^{P_{1}}+\cdots+\int_{\infty}^{P_{g}}\right)\left(\frac{1}{2 y}, \frac{x}{2 y}, \ldots, \frac{x^{g-1}}{2 y}\right) \mathrm{d} x
$$


of the first kind for $P_{j} \in C$. Let $\Lambda \subset C^{g}$ be the lattice of their periods. So $\boldsymbol{C}^{g} / \Lambda$ is the Jacobian variety of $C$. We denote the canonical map by $\kappa: \boldsymbol{C}^{g} \rightarrow \boldsymbol{C}^{g} / \Lambda$. We have an embedding $\iota: C \hookrightarrow \boldsymbol{C}^{g} / \Lambda$ defined by

$$
P \mapsto\left(\int_{\infty}^{P} \frac{\mathrm{d} x}{2 y}, \int_{\infty}^{P} \frac{x \mathrm{~d} x}{2 y}, \ldots, \int_{\infty}^{P} \frac{x^{g-1} \mathrm{~d} x}{2 y}\right) \bmod \Lambda .
$$

Therefore, $\iota(\infty)=(0,0, \ldots, 0) \in \boldsymbol{C}^{g} / \Lambda$. We regard an algebraic function on $C$, which we call a hyperelliptic function in this article, as a function on a universal Abelian covering $\kappa^{-1} \iota(C)\left(\subset C^{g}\right)$ of $C$. If $u=\left(u_{1}, \ldots, u_{g}\right)$ is in $\kappa^{-1} \iota(C)$, we denote by $(x(u), y(u))$ the coordinate of the corresponding point on $C$ by

$$
u=\int_{\infty}^{(x(u), y(u))}\left(\frac{1}{2 y}, \frac{x}{2 y}, \ldots, \frac{x^{g-1}}{2 y}\right) \mathrm{d} x
$$

with appropriate choice of a path for the integrals. Needless to say, we have $(x(0,0, \ldots, 0)$, $y(0,0, \ldots, 0))=\infty$.

Our new point of view is characterized by the following three features of the formulae (1.3) and (1.4). Firstly, the sequence of functions of $u$ whose values at $u=u^{(j)}$ are displayed in the $j$ th row of the determinant of (1.3) is just a sequence of the monomials of $x(u)$ and $y(u)$ displayed according to the order of their poles at $u=0$. Secondly, the two sides of (1.3) as a function of $u=u^{(j)}$ and those of (1.4) should be regarded as functions defined on the universal (Abelian) covering space $\boldsymbol{C}$ not of the Jacobian variety but of the elliptic curve. Thirdly, the expression of the left-hand side of (1.4) states that the function on the two sides of (1.4) is characterized as an elliptic function such that its zeros are exactly the points different from $\infty$ whose $n$-plication is just on the standard theta divisor in the Jacobian of the curve, and such that its only pole is at $\infty$. In the case of the elliptic curve above, the standard theta divisor is just the point at infinity.

Surprisingly enough, these three features can be used to derive a natural generalization of these formulae for hyperelliptic curves. More concretely, for $n \geqslant g$ our generalization of (1.4) is obtained by replacing the sequence giving the rows of the right-hand side by the sequence

$$
1, x(u), x^{2}(u), \ldots, x^{g}(u), y(u), x^{g+1}(u), y x(u), \ldots
$$

Here $u=\left(u_{1}, u_{2}, \ldots, u_{g}\right)$ is on $\kappa^{-1} \iota(C)$, with the monomials of $x(u)$ and $y(u)$ displayed according to the order of their poles at $u=(0,0, \ldots, 0)$, replacing the derivatives with respect to $u \in C$ by those with respect to $u_{1}$ along $\kappa^{-1} \iota(C)$. The left-hand side of (1.4) is replaced by

$$
\pm 1 ! 2 ! \cdots(n-1) ! \sigma(n u) / \sigma_{\sharp}(u)^{n^{2}},
$$

where $n \geqslant g, \sigma(u)=\sigma\left(u_{1}, u_{2}, \ldots, u_{g}\right)$ is a well-tuned Riemann theta series, which is a natural generalization of the classical $\sigma(u)$. The function $\sigma_{\sharp}$ is defined in the table below:

\begin{tabular}{c|ccccccccc} 
genus $g$ & 1 & 2 & 3 & 4 & 5 & 6 & 7 & 8 & $\cdots$ \\
\hline$\sigma_{\sharp}$ & $\sigma$ & $\sigma_{2}$ & $\sigma_{2}$ & $\sigma_{24}$ & $\sigma_{24}$ & $\sigma_{246}$ & $\sigma_{246}$ & $\sigma_{2468}$ & $\cdots$
\end{tabular}


where

$$
\sigma_{i j \cdots \ell}(u)=\frac{\partial}{\partial u_{i}} \frac{\partial}{\partial u_{j}} \cdots \frac{\partial}{\partial u_{\ell}} \sigma(u) .
$$

The function (1.5) is a natural generalization of the $n$-division polynomial of an elliptic curve, as mentioned in Remark 9.4 below. For the case $n \leqslant g$, we need a slight modification as in Theorems 8.2(i) and 9.3(i). As a function on $\kappa^{-1} \iota(C), \sigma_{\sharp}(u)$ has its zeros only at the points $\kappa^{-1} \iota(\infty)$ (Proposition 7.5(i)). This property is exactly the same as for the classical $\sigma(u)$. The hyperelliptic function (1.5) can be regarded as a function on $\boldsymbol{C}^{g}$ via theta functions. Although this function on $\boldsymbol{C}^{g}$ is no longer a function on the Jacobian, it is indeed expressed simply in terms of theta functions and is treated in a very similar way to that of elliptic functions.

The most difficult problem was to find the left-hand side of our expected generalization of (1.3). For simplicity we assume $n \geqslant g$. The answer is remarkably elegant and is

$$
\frac{\sigma\left(u^{(1)}+u^{(2)}+\cdots+u^{(n)}\right) \prod_{i<j} \sigma_{b}\left(u^{(i)}-u^{(j)}\right)}{\sigma_{\sharp}\left(u^{(1)}\right)^{n} \sigma_{\sharp}\left(u^{(2)}\right)^{n} \cdots \sigma_{\sharp}\left(u^{(n)}\right)^{n}},
$$

where $u^{(j)}=\left(u_{1}^{(j)}, u_{2}^{(j)}, \ldots, u_{g}^{(j)}\right)(j=1, \ldots, n)$ are variables on $\kappa^{-1} \iota(C)$ and $\sigma_{\mathrm{b}}(u)$ is defined as in the table below:

\begin{tabular}{c|ccccccccc} 
genus $g$ & 1 & 2 & 3 & 4 & 5 & 6 & 7 & 8 & $\cdots$ \\
\hline$\sigma_{b}$ & $\sigma$ & $\sigma$ & $\sigma_{3}$ & $\sigma_{3}$ & $\sigma_{35}$ & $\sigma_{35}$ & $\sigma_{357}$ & $\sigma_{357}$ & $\cdots$
\end{tabular}

Once we found this, we could prove the formula by, roughly speaking, comparing the divisors of the two sides. As the formula (1.4) is obtained by a limiting process from (1.3), our generalization of (1.4) is obtained by a similar limiting process from the generalization of (1.3).

Cantor [8] gave another determinant expression of the function that is characterized in the third feature explained above, for any hyperelliptic curve. The expression of Cantor should be seen as a generalization of a formula due to Brioschi (see [4, p. 770, 1. 3]).

Concerning the paper [19], Matsutani pointed out that (1.4) can be generalized to all hyperelliptic curves, and he proved that the resulting formula is equivalent to Cantor's one. He kindly permitted the author to include his proof as an appendix to this paper.

Matsutani's observation stimulated the author to start working on an extension of (1.3) for all hyperelliptic curves. The method of this paper is entirely different from that of $[\mathbf{1 9}, \mathbf{2 0}]$. It gives probably one of the simplest approaches to these extensions, and is based on the paper of Buchstaber, Enolskii and Leykin [6]. At the beginning of this research the author computed several cases by the 'low blow' method as in $[\mathbf{1 9}, \mathbf{2 0}]$. While Theorem 8.2 was still a conjecture Enolskii suggested to the author that to prove the conjecture it would be important to investigate the leading terms of the sigma function as in $[6]$.

Now we outline the idea of the proof. When the curve $C$, defined by $y^{2}=f(x)$, deforms to a singular curve $y^{2}=x^{2 g+1}$ the canonical limit of the function $\sigma(u)$ is known to be a Schur polynomial from the theory of representations of symmetric groups. The 
paper [6] treated this fact quite explicitly. Such a limit polynomial is called the SchurWeierstrass polynomial in that paper. For our argument, we need a slight extension of this fact (see $\S 5$ ). To prove our formula of Frobenius-Stickelberger type by induction on the number of variables $u^{(j)}$, we need relations to connect each factor of the numerator to a factor of the denominator in the left-hand side of the formula in Theorem 8.2. So, after proving such a connection with the Schur-Weierstrass polynomial as explained in $\S 3$, we will lift the connection to the case of the sigma function as in $\S 7$. For this, we need additional facts on the vanishing of some derivatives of the sigma function, as is described in $\S 6$.

The results from $\S 2$ to $\S 7$ are easily generalized to quite a wide family of algebraic curves. Such curves are called $(n, s)$-curves in $[\mathbf{6}, \mathbf{7}]$. Unfortunately the standard theta divisor or every standard theta subvariety, i.e. an image of the symmetric product of some copies of the curve, in the case of such a general curve, is not symmetric in the Jacobian. Here the word 'standard' means that the embedding of the curve into its Jacobian variety sends the point at infinity to the origin. Hence the sigma function of such a general curve has no involution and our naive generalization ended in failure.

There are also various generalizations of (1.1) (or (1.3)) in the case of genus two, different from our line of approach. An interested reader should consult the Introduction of $[\mathbf{1 9}]$.

\subsection{Notation}

We use the following notation throughout the paper. We denote, as usual, by $\boldsymbol{Z}, \boldsymbol{Q}$, $\boldsymbol{R}$ and $\boldsymbol{C}$ the ring of rational integers, the field of rational numbers, the field of real numbers and the field of complex numbers, respectively. In an expression for the Laurent expansion of a function, the symbol $\left(d^{\circ}\left(z_{1}, z_{2}, \ldots, z_{m}\right) \geqslant n\right)$ stands for the terms of total degree at least $n$ with respect to the variables $z_{1}, z_{2}, \ldots, z_{m}$. This notation never means that the terms are monomials only of $z_{1}, \ldots, z_{m}$. When the variable or the least total degree is clear from the context, we simply denote them by $\left(d^{\circ} \geqslant n\right)$ or the dots ' $\ldots$ '. We will often omit zero entries from a matrix. For simplicity we will occasionally denote an unspecified matrix entry with an asterisk.

\section{The Schur-Weierstrass polynomial}

We begin with a review of fundamentals on Schur-Weierstrass polynomials. Our main references are $[\mathbf{6}, \mathbf{1 5}]$.

Let $g$ be a fixed positive integer, and $u_{g}^{(1)}, \ldots, u_{g}^{(g)}$ be indeterminates. We fix $n(0 \leqslant$ $n \leqslant g)$ and we denote by $\boldsymbol{u}_{g}$ the set of variables $u_{g}^{(1)}, \ldots, u_{g}^{(n)}$. For each $k \geqslant 0$ we denote by $(-1)^{k} U_{k}^{[n]}\left(\boldsymbol{u}_{g}\right)$ the $k$ th complete symmetric function, namely the sum of all monomials of total degree $k$ of the variables $u_{g}^{(1)}, \ldots, u_{g}^{(n)}$. We will emphasize by the superscript ' $[n]$ ' that $U_{k}^{[n]}\left(\boldsymbol{u}_{g}\right)$ is a function of a set of $n$ variables $\boldsymbol{u}_{g}$. If $k<0$, then we regard $U_{k}^{[n]}\left(\boldsymbol{u}_{g}\right)$ as 0 .

We now consider the determinant

$$
\left|U_{g-2 i+j+1}^{[g]}\left(\boldsymbol{u}_{g}\right)\right|_{1 \leqslant i, j \leqslant g} .
$$


If we write simply $U_{k}=U_{k}^{[g]}\left(\boldsymbol{u}_{g}\right)$, this is explicitly of the form

$$
\left|\begin{array}{cccccc}
U_{g} & U_{g+1} & U_{g+2} & \cdots & U_{2 g-2} & U_{2 g-1} \\
U_{g-2} & U_{g-1} & U_{g} & \cdots & U_{2 g-4} & U_{2 g-3} \\
\vdots & \vdots & \vdots & \ddots & \vdots & \vdots \\
U_{1} & U_{2} & U_{3} & \cdots & * & * \\
& U_{0} & U_{1} & \cdots & * & * \\
& & & \ddots & \vdots & \vdots \\
& & & & U_{0} & U_{1}
\end{array}\right|
$$

for odd $g$, or

$$
\left|\begin{array}{ccccccc}
U_{g} & U_{g+1} & U_{g+2} & U_{g+3} & \cdots & U_{2 g-2} & U_{2 g-1} \\
U_{g-2} & U_{g-1} & U_{g} & U_{g+1} & \cdots & U_{2 g-4} & U_{2 g-3} \\
\vdots & \vdots & \vdots & \vdots & \ddots & \vdots & \vdots \\
U_{0} & U_{1} & U_{2} & U_{3} & \cdots & * & * \\
& & U_{0} & U_{1} & \cdots & * & * \\
& & & & \ddots & \vdots & \vdots \\
& & & & & U_{0} & U_{1}
\end{array}\right|
$$

for even $g$.

We make use of the following definitions in the rest of the paper:

$$
\begin{aligned}
p_{j} & :=\left(u_{g}^{(1)}\right)^{j}+\cdots+\left(u_{g}^{(g)}\right)^{j}, \\
u_{j}^{(i)} & :=\frac{1}{2(g-j)+1}\left(u_{g}^{(i)}\right)^{2(g-j)+1}, \\
u^{(i)} & :=\left(u_{1}^{(i)}, \ldots, u_{g}^{(i)}\right), \\
u_{j} & :=u_{j}^{(1)}+u_{j}^{(2)}+\cdots+u_{j}^{(g)}=\frac{1}{2(g-j)+1} p_{2(g-j)+1}, \\
u & :=u^{(1)}+u^{(2)}+\cdots+u^{(g)}=\left(u_{1}, u_{2}, \ldots, u_{g}\right) .
\end{aligned}
$$

In fact our $\left|U_{g-2 i+j+1}^{[g]}\left(\boldsymbol{u}_{g}\right)\right|_{1 \leqslant i, j \leqslant g}$ is none other than $S_{2,2 g+1}$ in $[\mathbf{6}]$. We introduce new variables $s_{1}, s_{2}, \ldots, s_{2 g-1}$ satisfying

$$
p_{j}=-s_{1}{ }^{j}-s_{2}^{j}-\cdots-s_{2 g-1}{ }^{j} \quad(1 \leqslant j \leqslant 2 g-1) .
$$

Such $s_{j}$ exist for the following reason. Denoting by $\varepsilon_{k}(\boldsymbol{s})$ with $\boldsymbol{s}=\left(s_{1}, s_{2}, \ldots, s_{2 g-1}\right)$ the elementary symmetric function of degree $k$ of $s_{j}$, the numbers $s_{1}, s_{2}, \ldots, s_{2 g-1}$ satisfying (2.3) are solutions of

$$
\varepsilon_{k}(\boldsymbol{s})=\frac{1}{k !}\left|\begin{array}{ccccc}
-p_{1} & 1 & & & \\
-p_{2} & -p_{1} & 2 & & \\
\vdots & \vdots & \vdots & \ddots & \\
-p_{k-1} & -p_{k-2} & -p_{k-3} & \cdots & k-1 \\
-p_{k} & -p_{k-1} & -p_{k-2} & \cdots & -p_{1}
\end{array}\right|=(-1)^{k} U_{k}^{[g]}\left(\boldsymbol{u}_{g}\right)
$$


for $k=1,2, \ldots, 2 g-1$. This fact is given by [15, p. 29, 1. -4 , and p. 28, 1. 13]. The fundamental theorem of the algebraic equations shows that such a system of equations always has solutions. Hence, we see that $\left|U_{k}^{[g]}\left(\boldsymbol{u}_{g}\right)\right|$ coincides with the Schur-Weierstrass polynomial $S_{2,2 g+1}\left(-p_{1},-p_{3}, \ldots,-p_{2 g-1}\right)$ of Theorem 4.3 in [6].

Remark 2.1. Here note that our $u_{j}$ are slightly different from the $z_{j}$ of [6]. The correspondence is given by $z_{j}=-(2 j-1) u_{g-j+1}$. Moreover, it would be helpful for the reader to understand the relationship between our setting and that of [6]. Compare the integral (4.2) below with the definition (5.3) of [6], which has a multiplicative constant and integrals in the opposite direction.

While $\left|U_{g-2 i+j+1}^{[g]}\left(\boldsymbol{u}_{g}\right)\right|$ is obviously a polynomial of $u_{g}^{(1)}, \ldots, u_{g}^{(g)}$, we can prove more.

Proposition 2.2. The polynomial

$$
\left|U_{g-2 i+j+1}^{[g]}\left(\boldsymbol{u}_{g}\right)\right|_{1 \leqslant i, j \leqslant g}
$$

above is completely determined by the $g$ values of $-p_{1},-p_{3}, \ldots,-p_{2 g-1}$ defined above, and so also by $u_{1}, u_{2}, \ldots, u_{g}$.

Proof. See Theorem 4.1 in [6, p. 86].

So we may write

$$
S(u):=\left|U_{g-2 i+j+1}^{[g]}\left(\boldsymbol{u}_{g}\right)\right|_{1 \leqslant i, j \leqslant g} .
$$

This polynomial $S(u)$ is called the Schur-Weierstrass polynomial.

We introduce a polynomial weight, defined by taking the weight of $u_{j}$ as being $2(g-j)+1$. We call this the Sato weight. It is easy to see that $S(u)$ is homogeneous with respect to the Sato weight, and has the Sato weight $\frac{1}{2} g(g+1)$.

Let $m$ be a fixed positive integer and $\xi_{1}, \ldots, \xi_{m}$ be indeterminates. We denote $(-1)^{k}$ times the sum of monomials of total degree $k$ of $\xi_{1}, \ldots, \xi_{m}$ by $U_{k}^{[m]}(\xi)$ as above, where $\xi$ means the set of $\xi_{1}, \ldots, \xi_{m}$.

Definition 2.3. Let $m, \xi_{i}$ and $U_{k}^{[m]}(\xi)$ be as above. A matrix, each of whose rows consists of $(m+1)$ successive terms of the sequence

$$
\ldots, 0,0,1, U_{1}^{[m]}(\xi), U_{2}^{[m]}(\xi), \ldots
$$

except the set of terms

$$
0, \ldots, 0,0,1
$$

is called a fundamental matrix without a simple row with respect to $\xi_{1}, \ldots, \xi_{m}$.

The following is used several times in $\S 3$.

Lemma 2.4. Let $m, \xi_{i}$ and $U_{k}^{[m]}(\xi)$ be as above, and $M$ be a fundamental matrix without a simple row with respect to $\xi_{1}, \ldots, \xi_{m}$. We denote by $\varepsilon_{j}(\xi)$ the elementary 
symmetric function of $\xi_{1}, \ldots, \xi_{m}$ of degree $j$. Then we have

$$
M\left[\begin{array}{c}
\varepsilon_{m}(\xi) \\
\varepsilon_{m-1}(\xi) \\
\vdots \\
\varepsilon_{1}(\xi) \\
1
\end{array}\right]=\left[\begin{array}{c}
0 \\
0 \\
\vdots \\
0 \\
0
\end{array}\right] .
$$

Proof. See [15, p. 21, $\left.\left(2.6^{\prime}\right)\right]$.

Although the following will be not used in this paper explicitly, it is deeply related to the Riemann singularity theorem, which is mentioned in $\S 6$. So we give it here.

Lemma 2.5. As a polynomial in $u_{g}^{(1)}, \ldots, u_{g}^{(g-1)}, u_{g}^{(g)}$, we have

$$
S\left(u^{(1)}+\cdots+u^{(g-1)}+u^{(g)}\right)=0
$$

identically when $u_{g}^{(g)}=0$.

Proof. This formula follows from Lemma 2.4 by setting $m=g-1$ and $M$ to be the matrix whose determinant expresses $S\left(u^{(1)}+\cdots+u^{(g-1)}\right)$.

\section{Derivatives of the Schur-Weierstrass polynomial}

We will discuss some derivatives of the Schur-Weierstrass polynomial in order to investigate the corresponding derivatives of the sigma function in $\S 7$.

Definition 3.1. For an integer $n$ with $1 \leqslant n \leqslant g$, we denote by $\natural^{n}$ the set of positive integers $i$ such that $n+1 \leqslant i \leqslant g$ with $i \equiv n+1 \bmod 2$. Explicitly, $\natural^{n}$ is the set $\{n+1, n+3, \ldots, g-3, g-1\}$ or $\{n+1, n+3, \ldots, g-2, g\}$ according to whether $n \equiv g \bmod 2$ or not.

Definition 3.2. We denote by $S_{\natural n}(u)$ the derivative

$$
\left(\prod_{i \in \mathfrak{\natural}^{n}} \frac{\partial}{\partial u_{i}}\right) S(u)
$$

We define in particular $\sharp=\natural^{1}$ and $b=\natural^{2}$, so that $S_{\sharp}(u)=S_{\mathfrak{\natural}^{1}}(u)$ and $S_{b}(u)=S_{\mathfrak{\natural}^{2}}(u)$.

In this section we define, as in $\S 2$,

$$
p_{k}:=\sum_{i=1}^{g}\left(u_{g}^{(i)}\right)^{k} \quad \text { and } \quad u_{j}=\frac{1}{2 g-2 j+1} p_{2 g-2 j+1} \quad \text { for } j=1, \ldots, g .
$$

Lemma 3.3. If we regard a polynomial of $U_{1}^{[g]}\left(\boldsymbol{u}_{g}\right), U_{2}^{[g]}\left(\boldsymbol{u}_{g}\right), \ldots$ as a polynomial in $p_{1}, \ldots, p_{2 g-1}$, we then have

$$
k \frac{\partial}{\partial p_{k}}=(-1)^{k} \sum_{r \geqslant 0} U_{r} \frac{\partial}{\partial U_{k+r}},
$$

where we simply write $U_{j}=U_{j}^{[g]}\left(\boldsymbol{u}_{g}\right)$. 
Proof. See [15, p. 76].

Now we continue to write $U_{j}=U_{j}^{[g]}\left(\boldsymbol{u}_{g}\right)$. The formula states that $(-1)^{k} k\left(\partial / \partial p_{k}\right) S(u)$ is the sum of the determinants obtained by 'shifting by $k$ ' one of the rows towards the right of the matrix of the determinant expression of $S(u)$.

Proposition 3.4. Let $n$ be an integer such that $1 \leqslant n \leqslant g-1$ and let $v_{g}$ be a scalar variable. Let

$$
\begin{aligned}
v & =\left(\frac{1}{2 g-1} v_{g}^{2 g-1}, \ldots, \frac{1}{3} v_{g}^{3}, v_{g}\right), \\
u^{(j)} & =\left(\frac{1}{2 g-1}\left(u_{g}^{(j)}\right)^{2 g-1}, \ldots, \frac{1}{3}\left(u_{g}^{(j)}\right)^{3}, u_{g}^{(j)}\right) .
\end{aligned}
$$

Then

(i) $S_{\sharp}(v)=-(-1)^{(g-1)(g-2)(g-3) / 2} v_{g}^{g}$;

(ii) $S_{\mathfrak{\natural n}^{n+1}}\left(u^{(1)}+\cdots+u^{(n)}+v\right)$

$$
=(-1)^{(g-n)(g-n-1) / 2} S_{\mathfrak{\natural}^{n}}\left(u^{(1)}+\cdots+u^{(n)}\right) v^{g-n}+\left(d^{\circ}\left(v_{g}\right) \geqslant g-n+2\right) ;
$$

(iii) $S_{b}(2 v)=-(-1)^{g(g-1)(g-2) / 2} 2 v_{g}^{2 g-1}$.

We first prove (ii), then (i), and finally (iii).

Proof of (ii). While we consider four cases according to the parity of $g$ and $n$, all the cases are proved similarly. We see that

$$
\natural^{n+1}= \begin{cases}\{n+2, n+4, \ldots, g-2, g\} & \text { if } n \equiv g \bmod 2, \\ \{n+2, n+4, \ldots, g-3, g-1\} & \text { if } n \not \equiv g \bmod 2 .\end{cases}
$$

We denote the number of this set by $\nu$ :

$$
\nu= \begin{cases}(g-n) / 2 & \text { if } n \equiv g \bmod 2, \\ (g-n-1) / 2 & \text { if } n \neq \equiv g \bmod 2 .\end{cases}
$$

The expansion that we are concerned with is the derivative $S_{\mathrm{t}^{n+1}}(u)$ of $S(u)$, which is a function of $g$ variables $u=\left(u_{1}, \ldots, u_{g}\right)$ to be substituted later by $u=u^{(1)}+\cdots+u^{(n)}+v$. We will apply

$$
\begin{aligned}
D_{n+1}= & \prod_{i \in \mathfrak{\natural}^{n+1}} \frac{\partial}{\partial u_{i}} \\
= & \left\{\begin{array}{cc}
(2 g-2 n-3) \frac{\partial}{\partial p_{2 g-2 n-3}}(2 g-2 n-7) \frac{\partial}{\partial p_{2 g-2 n-7}} \cdots 5 \frac{\partial}{\partial p_{5}} 1 \frac{\partial}{\partial p_{1}} & \text { (if } n \equiv g \bmod 2), \\
(2 g-2 n-3) \frac{\partial}{\partial p_{2 g-2 n-3}}(2 g-2 n-7) \frac{\partial}{\partial p_{2 g-2 n-7}} \cdots & 7 \frac{\partial}{\partial p_{7}} 3 \frac{\partial}{\partial p_{3}} \\
\text { (if } n \neq \equiv \bmod 2)
\end{array}\right.
\end{aligned}
$$


to $S(u)$. Using the formula in Lemma 3.3 and expressing $S(u)$ as the determinant

$$
\left|U_{g-2 i+j+1}\right|_{1 \leqslant i, j \leqslant g} \quad \text { with } U_{k}=U_{k}^{[g]}\left(u^{(1)}, \ldots, u^{(g)}\right)
$$

show explicitly the result given by applying $D_{n+1}$ to $S(u)$. Namely, each of the factors of $D_{n+1}$ in (3.1) is simply the sum of certain shifting to the right of all rows of $S(u)=$ $\left|U_{g-2 i+j+1}\right|$. To explain this more concretely, we regard each row of $S(u)$ to be a partial sequence consisting successive $g$ terms of the two-sided infinite sequence

$$
\ldots, 0,0, \ldots, 0, U_{0}, U_{1}, U_{2}, \ldots
$$

We denote by $\operatorname{sh}_{i}^{j}$ the operation of shifting by $j$ terms to the right on the $i$ th row. For example, if the $i$ th row is $\left(U_{2}, U_{3}, U_{4}, U_{5}, \ldots, U_{g+1}\right)$, then $\operatorname{sh}_{i}^{3}$ transforms this row to $\left(0, U_{0}, U_{1}, U_{2}, \ldots, U_{g-2}\right)$. Then we have

$$
\begin{aligned}
S_{\mathfrak{\natural}^{n+1}}(u) & =D_{n+1} S(u) \\
& =\gamma_{n+1}^{\prime} \times \begin{cases}\sum_{1 \leqslant i_{1}, \ldots, i_{\nu} \leqslant g} \operatorname{sh}_{i_{1}}^{4 \nu-1} \operatorname{sh}_{i_{2}}^{4 \nu-5} \cdots \operatorname{sh}_{i_{\nu-1}}^{5} \operatorname{sh}_{i_{\nu}}^{1}\left|U_{g-2 i+j+1}\right| & \text { if } n \equiv g \bmod 2, \\
\sum_{1 \leqslant i_{1}, \ldots, i_{\nu} \leqslant g} \operatorname{sh}_{i_{1}}^{4 \nu-1} \operatorname{sh}_{i_{2}}^{4 \nu-5} \cdots \operatorname{sh}_{i_{\nu-1}}^{7} \operatorname{sh}_{i_{\nu}}^{3}\left|U_{g-2 i+j+1}\right| & \text { if } n \not \equiv g \bmod 2 .\end{cases}
\end{aligned}
$$

Here $\gamma_{n+1}^{\prime}$ is a signature \pm 1 coming from the top of the formula in Lemma 3.3 , and is given by

$$
\gamma_{n+1}^{\prime}= \begin{cases}(-1)^{(g-n+1)(g-n) / 2} & \text { if } n \equiv g \bmod 2 \\ (-1)^{(g-n)(g-n-1) / 2} & \text { if } n \not \equiv g \bmod 2 .\end{cases}
$$

We claim that if we put

$$
u=u^{(1)}+\cdots+u^{(n)}+v
$$

in (3.2), namely, if we set $U_{g-2 i+j+1}$ to be

$$
U_{g-2 i+j+1}^{[n+1]}=U_{g-2 i+j+1}^{[n+1]}\left(u_{g}^{(1)}, \ldots, u_{g}^{(n)}, v_{g}\right),
$$

then all the terms in (3.2) vanish except one. The unique non-vanishing term is obtained from

$$
\gamma_{n+1}^{\prime}|M|:=\gamma_{n+1}^{\prime} \times \begin{cases}\operatorname{sh}_{n+1}^{4 \nu-1} \operatorname{sh}_{n+2}^{4 \nu-5} \cdots \operatorname{sh}_{n+\nu-1}^{5} \operatorname{sh}_{n+\nu}^{1}\left|U_{g-2 i+j+1}\right| & \text { if } n \equiv g \bmod 2, \\ \operatorname{sh}_{n+1}^{4 \nu-1} \operatorname{sh}_{n+2}^{4 \nu-5} \cdots \operatorname{sh}_{n+\nu-1}^{7} \operatorname{sh}_{n+\nu}^{3}\left|U_{g-2 i+j+1}\right| & \text { if } n \not \equiv g \bmod 2\end{cases}
$$


by putting $u=u^{(1)}+\cdots+u^{(n)}$. We denote it by

$$
\gamma_{n+1}^{\prime}\left|M^{[n+1]}\right|
$$

Here the determinant $|M|$ is expressed as follows: if $n \equiv g \bmod 2$, then

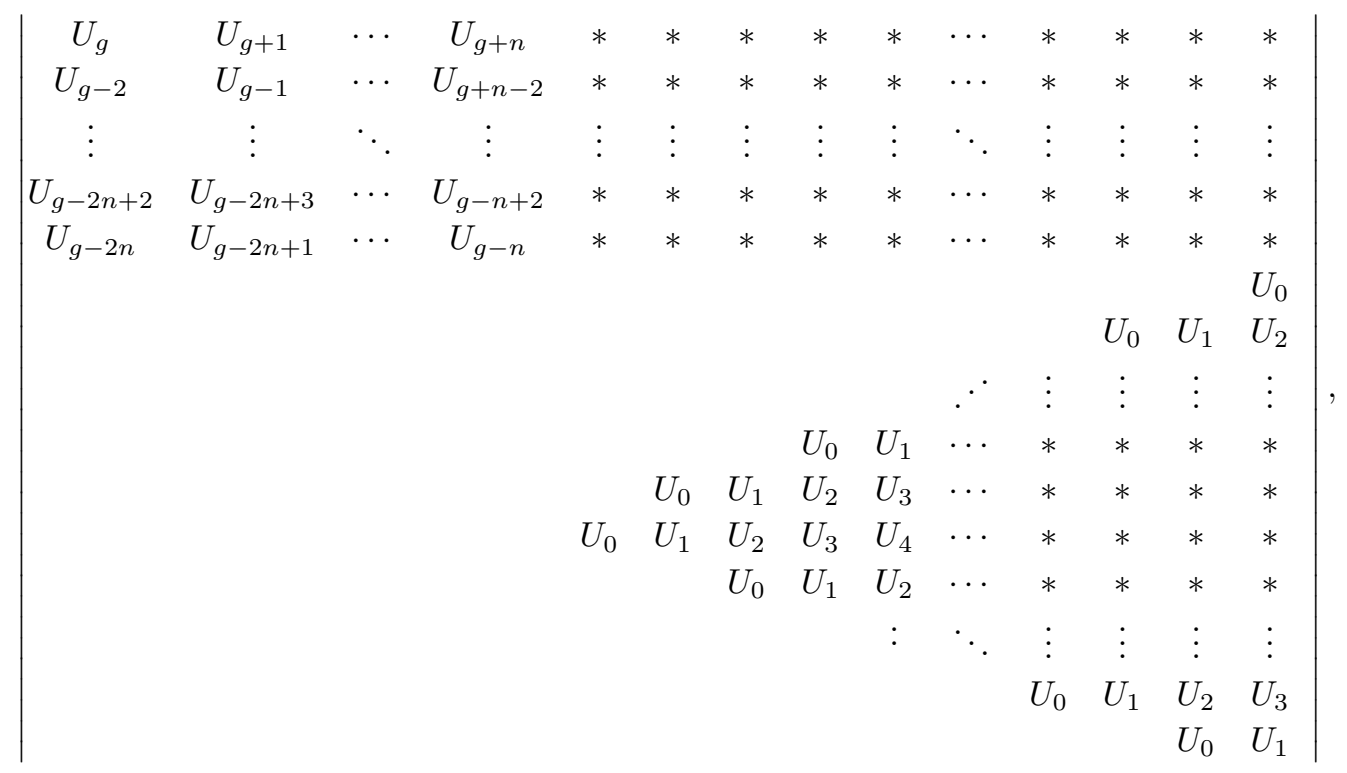

and, if $n \not \equiv g \bmod 2$, then

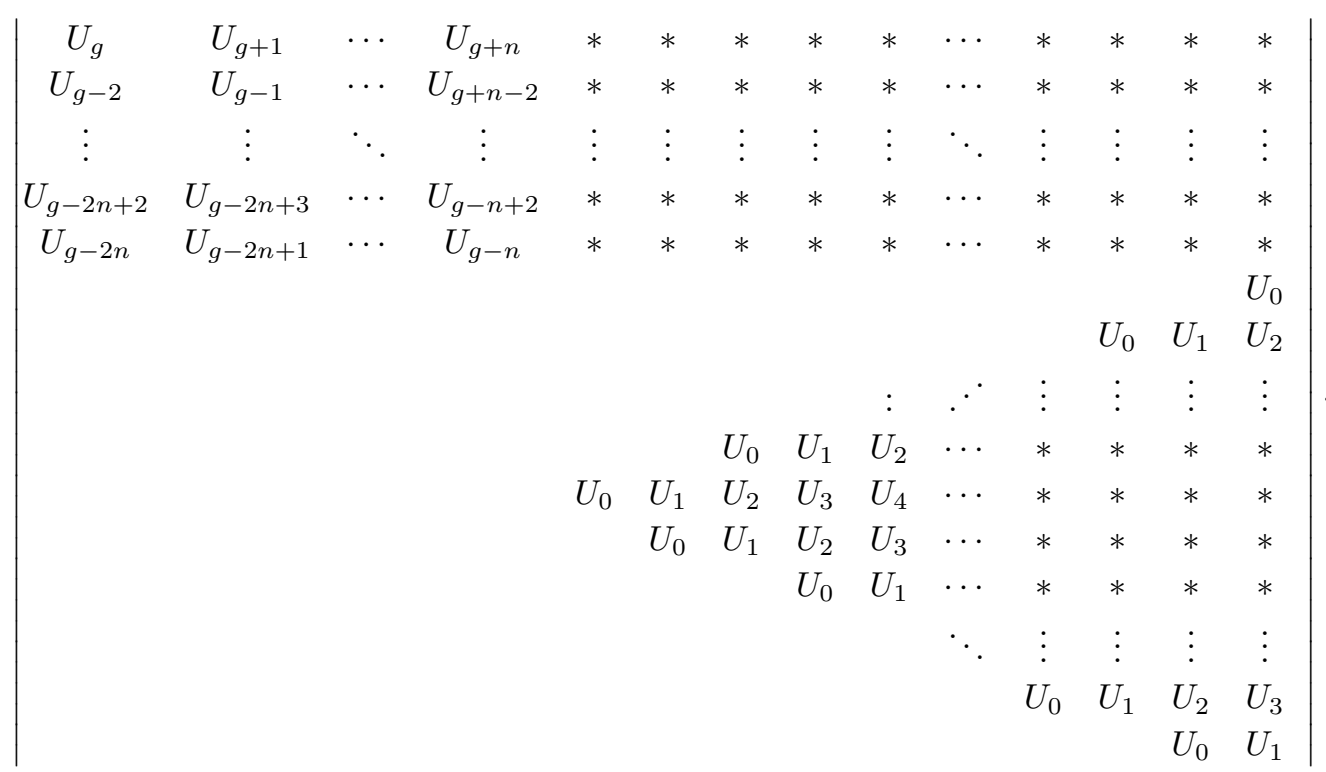


In both cases, each determinant is obviously transformed to

$$
\gamma_{n+1}^{\prime \prime}\left|\begin{array}{cccccccc}
U_{g} & U_{g+1} & \cdots & U_{g+n} & * & * & \cdots & * \\
U_{g-2} & U_{g-1} & \cdots & U_{g+n-2} & * & * & \cdots & * \\
\vdots & \vdots & \ddots & \vdots & \vdots & \vdots & \ddots & \vdots \\
U_{g-2 n+2} & U_{g-2 n+3} & \cdots & U_{g-n+2} & * & * & \cdots & * \\
U_{g-2 n} & U_{g-2 n+1} & \cdots & U_{g-n} & * & * & \cdots & * \\
& & & & U_{0} & * & \cdots & * \\
& & & & & U_{0} & \cdots & * \\
& & & & & & \ddots & \vdots \\
& & & & & & & U_{0}
\end{array}\right|,
$$

where $\gamma_{n+1}^{\prime \prime}$ is the signature coming from exchanges of rows and is given by

$$
\gamma_{n+1}^{\prime \prime}= \begin{cases}1 & \text { if } g-(n+1) \not \equiv 2 \bmod 4, \\ -1 & \text { if } g-(n+1) \equiv 2 \bmod 4 .\end{cases}
$$

We will now prove the claim above by the following three steps.

Step 1. If the index $\left(i_{1}, \ldots, i_{\nu}\right)$ corresponding to a term in (3.2) contains a repetition, then this term vanishes when we put $u=u^{(1)}+\cdots+u^{(n)}$, for the following reason. Since $j$ of $\operatorname{sh}_{i}^{j}$ is always odd, any two shifts in the same row is a shift by an even number of terms. By looking at the expression $(2.1 a)$ and $(2.1 b)$ of $\left|U_{g-2 i+j+1}\right|$, we see that either the shifted row coincides with another row or all the terms in the row are 0 , and such a determinant vanishes before applying the remaining shifts and substituting $u=u^{(1)}+\cdots+u^{(n)}$. Hence, in this case, no non-zero term appears.

Step 2. In this step, any $g \times g$ matrix $\left[a_{i j}\right]$ whose determinant we will consider is regarded as being divided into four blocks according as $i \leqslant(n+1)$ or not, and as $j \leqslant(n+1)$ or not. We take an arbitrary term of the sum (3.2) (after all shifts have been applied). We reorder its rows so that any column is ordered by increasing index. We denote the resulting determinant by $\left|M_{1}\right|$, and by $\left|M_{1}^{[n+1]}\right|$ the determinant obtained from $\left|M_{1}\right|$ by putting $u=u^{(1)}+\cdots+u^{(n)}+v$. Suppose the region of $\left|M_{1}^{[n+1]}\right|$ consisting the $(n+1)$ th row and below contains at least one multi-step as

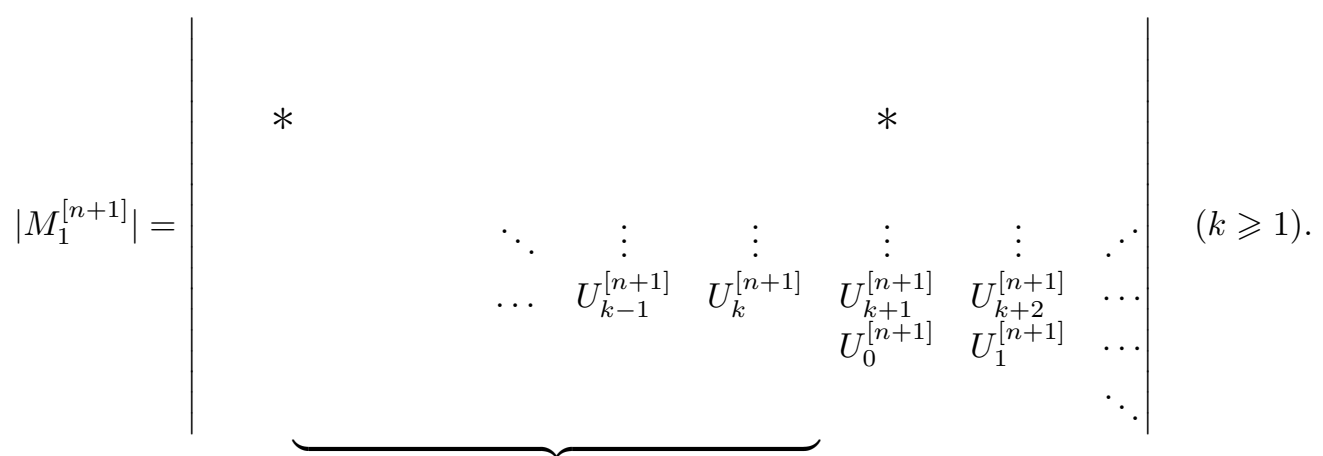

taking $n+2$ columns 
Then the successive $n+2$ columns, proceeding leftwards from the column that contains the multi-step above, form a matrix without simple rows. By Lemma 2.4 they are linearly dependent with respect to $n+1$ variables $u_{g}^{(1)}, \ldots, u_{g}^{(n)}, v_{g}$. Hence $\left|M_{1}^{[n+1]}\right|=0$. Therefore, we may consider determinants such that all their diagonals in the right-lower block are $U_{0}(=1)$.

Step 3. Since the totality of the shifts in every term in (3.2) is always the same, Step 2 states that we may consider only the terms in (3.2) such that the sum of the indices of the entries of the first column in the upper left block is equal to the same sum for $|M|$. Since we can shift only to the right, the first row of $|M|$ is none other than the first row of $S(u)$. Hence we may suppose that the sum of the indices from the $(2,1)$-entry to the $(n+1,1)$-entry in the first column is equal to that sum for $|M|$. Then the second row must be just the second row of $|M|$. Repeating these considerations, we conclude that no row in the upper blocks has undergone any $\operatorname{shift} \operatorname{sh}_{i}^{j}$. Hence we see that $\gamma_{n+1}^{\prime}\left|M^{[n+1]}\right|$ is the unique non-zero term in (3.2).

Summing up, we see that all the terms in (3.2) except $\gamma_{n+1}^{\prime}\left|M^{[n+1]}\right|$ vanish, so that we have

$$
\begin{aligned}
& S_{\mathfrak{\natural}^{n+1}}\left(u^{(1)}+\cdots+u^{(n)}+v\right) \\
& =\gamma_{n+1}\left|\begin{array}{ccccccccc}
U_{g}^{[n+1]} & U_{g+1}^{[n+1]} & \cdots & U_{g+n-1}^{[n+1]} & U_{g+n}^{[n+1]} & * & * & \cdots & * \\
U_{g-2}^{[n+1]} & U_{g-1}^{[n+1]} & \cdots & U_{g+n-3}^{[n+1]} & U_{g+n-2}^{[n+1]} & * & * & \cdots & * \\
\vdots & \vdots & \ddots & \vdots & \vdots & \vdots & \vdots & \ddots & \vdots \\
U_{g-2 n+2}^{[n+1]} & U_{g-2 n+3}^{[n+1]} & \cdots & U_{g-n+1}^{[n+1]} & U_{g-n+2}^{[n+1]} & * & * & \cdots & * \\
U_{g-2 n}^{[n+1]} & U_{g-2 n+1}^{[n+1]} & \cdots & U_{g-n-1}^{[n+1]} & U_{g-n}^{[n+1]} & * & * & \cdots & * \\
& & & & & 1 & * & \cdots & * \\
& & & & & & 1 & \cdots & * \\
& & & & & & \ddots & \vdots \\
& & & & & & & & \\
& & & & & & &
\end{array}\right|,
\end{aligned}
$$

where

$$
U_{k}^{[n+1]}=U_{k}^{[n+1]}\left(u_{1}^{(g)}, \ldots, u_{g}^{(g)}, v_{g}\right)
$$

and

$$
\gamma_{n+1}=\gamma_{n+1}^{\prime} \gamma_{n+1}^{\prime \prime}= \begin{cases}(+1)(+1)=1 & \text { if } g-n \equiv 0 \bmod 4, \\ (+1)(+1)=1 & \text { if } g-n \equiv 1 \bmod 4, \\ (-1)(+1)=-1 & \text { if } g-n \equiv 2 \bmod 4, \\ (-1)(-1)=1 & \text { if } g-n \equiv 3 \bmod 4 .\end{cases}
$$

Here we have used the fact that $U_{0}=1$. It is easily checked that

$$
\gamma_{n+1}=(-1)^{(g-n)(g-n-1)(g-n-3)} .
$$


Similarly, we have

$$
S_{\text {七n }}\left(u^{(1)}+\cdots+u^{(n)}\right)=\gamma_{n} \mid \begin{array}{cccccccc}
U_{g}^{[n]} & U_{g+1}^{[n]} & \cdots & U_{g+n-1}^{[n]} & * & * & \cdots & * \\
U_{g-2}^{[n]} & U_{g-1}^{[n]} & \cdots & U_{g+n-3}^{[n]} & * & * & \cdots & * \\
\vdots & \vdots & \ddots & \vdots & \vdots & \vdots & \ddots & \vdots \\
U_{g-2 n-2}^{[n]} & U_{g-2 n-1}^{[n]} & \cdots & U_{g-n+1}^{[n]} & * & * & \cdots & * \\
& & & & 1 & * & \cdots & * \\
& & & & & 1 & \cdots & * \\
& & & & & & \ddots & \vdots \\
& & & & & & & \\
& & & & & & &
\end{array}
$$

where $U_{k}^{[n]}=U_{k}^{[n]}\left(u_{1}^{(g)}, \ldots, u_{g}^{(g)}\right)$. For the determinant (3.3), we subtract $v_{g}$ times the $n$th column from the $(n+1)$ th column. The next time, we subtract $v_{g}$ times the $(n-1)$ th column from the $n$th column. Repeating such transformations for (3.3), we see that (3.3) is equal to

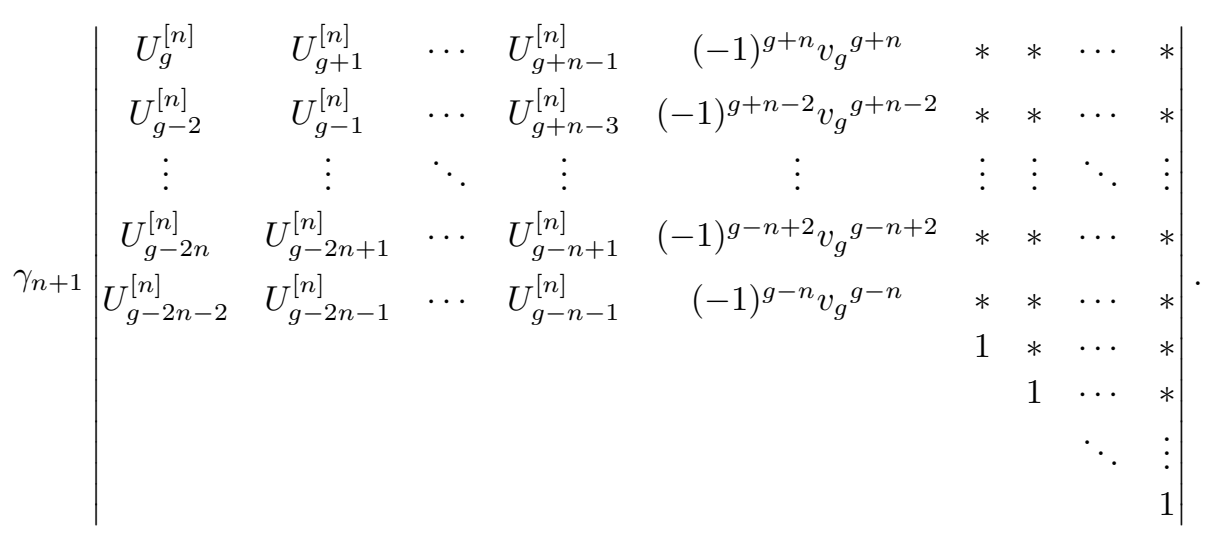

Expanding this by its $(n+1)$ th column and looking at (3.4) yields the expansion of (ii) up to a signature. The signature is obviously given by

$$
\gamma_{n+1} / \gamma_{n}= \begin{cases}(+1) /(+1)=1 & \text { if } g-n \equiv 0 \bmod 4 \\ (+1) /(-1)=-1 & \text { if } g-n \equiv 1 \bmod 4 \\ (-1) /(+1)=-1 & \text { if } g-n \equiv 2 \bmod 4 \\ (+1) /(+1)=1 & \text { if } g-n \equiv 3 \bmod 4\end{cases}
$$

times the signature of the $(n+1, n+1)$-entry of $(3.5)$, so that the signature is

$$
\gamma_{n+1} / \gamma_{n} \cdot(-1)^{g-n}=(-1)^{(g-n)(g-n-3) / 2}(-1)^{g-n}=(-1)^{(g-n)(g-n-1) / 2} .
$$

Thus we have proved (ii). 
Proof of (i). By (3.4), we have for

$$
v=\left(\frac{1}{2 g-1} v_{g}^{2 g-1}, \ldots, \frac{1}{3} v_{g}^{3}, v_{g}\right)
$$

that

$$
S_{\sharp}(v)=\gamma_{1}(-1)^{g} v_{g}^{g}=(-1)^{g(g-1)(g-3) / 2}(-1)^{g} v_{g}^{g}=-(-1)^{(g-1)(g-2)(g-3) / 2} v_{g}^{g} .
$$

Proof of (iii). For

$$
v=\left(\frac{1}{2 g-1} v_{g}^{2 g-1}, \ldots, \frac{1}{3} v_{g}^{3}, v_{g}\right)
$$

we have by (3.4) that

$$
\begin{aligned}
S_{\mathrm{b}}(2 v) & =\gamma_{2}\left(U_{g}^{[2]}(v, v) \cdot U_{g-1}^{[2]}(v, v)-U_{g-2}^{[2]}(v, v) \cdot U_{g+1}^{[2]}(v, v)\right) \\
& =(-1)^{(g-1)(g-2) g / 2}(-1)^{2 g-1}\left((g+1) v_{g}{ }^{g} \cdot g v_{g}{ }^{g-1}-(g-1) v_{g}{ }^{g-2} \cdot(g+2) v_{g}{ }^{g+1}\right) \\
& =-(-1)^{g(g-1)(g-2) / 2}((g+1) g-(g-1)(g+2)) v_{g}{ }^{2 g-1} \\
& =-(-1)^{g(g-1)(g-2) / 2} 2 v_{g}{ }^{2 g-1} .
\end{aligned}
$$

Now all the statements have been proved completely.

\section{Hyperelliptic functions}

In this section we recall the fundamentals of the theory of hyperelliptic functions.

Let $C$ be a smooth projective model of a curve of genus $g>0$ defined over $C$ whose affine equation is given by $y^{2}=f(x)$, where

$$
f(x)=\lambda_{0} x^{2 g+1}+\lambda_{1} x^{2 g}+\cdots+\lambda_{2 g} x+\lambda_{2 g+1} .
$$

In this paper, we always assume that $\lambda_{0}=1$. We will use, however, the symbol $\lambda_{0}$ too when this notation makes an equation of homogeneous weight easy to read.

We denote by $\infty$ the point of $C$ at infinity. It is known that the set of

$$
\omega_{j}:=\frac{x^{j-1} \mathrm{~d} x}{2 y} \quad(j=1, \ldots, g)
$$

forms a basis of the space of the differential forms of the first kind on $C$. As usual we let $\left[\omega^{\prime} \omega^{\prime \prime}\right]$ be the period matrix for a suitable choice of the basis of the fundamental group of $C$. Then the modulus of $C$ is given by $Z:=\omega^{-1} \omega^{\prime \prime}$. The lattice of periods is denoted by $\Lambda$, that is,

$$
\Lambda:=\omega^{\prime}\left[\begin{array}{llll}
\boldsymbol{Z} & \boldsymbol{Z} & \cdots & \boldsymbol{Z}
\end{array}\right]^{\mathrm{T}}+\omega^{\prime \prime}\left[\begin{array}{llll}
\boldsymbol{Z} & \boldsymbol{Z} & \cdots & \boldsymbol{Z}
\end{array}\right]^{\mathrm{T}} \quad\left(\subset \boldsymbol{C}^{g}\right) .
$$


Let

$$
\eta_{j}:=\frac{1}{2 y} \sum_{k=j}^{2 g-j}(k+1-j) \lambda_{2 g-k-j} x^{k} \mathrm{~d} x \quad(j=1, \ldots, g),
$$

which are differential forms of the second kind without poles except at $\infty$ (see [3, p. 195, Example i] or $\left[\mathbf{2}\right.$, p. 314]). We introduce the matrices of periods $\left[\begin{array}{ll}\eta^{\prime} & \eta^{\prime \prime}\end{array}\right]$ with respect to $\eta_{1}, \ldots, \eta_{g}$ for the basis of the fundamental group of $C$ chosen as above. We let

$$
\delta^{\prime \prime}:=\left[\begin{array}{llll}
\frac{1}{2} & \frac{1}{2} & \cdots & \frac{1}{2}
\end{array}\right]^{\mathrm{T}}, \quad \delta^{\prime}:=\left[\begin{array}{llll}
\frac{1}{2} g & \frac{1}{2}(g-1) & \cdots & \frac{1}{2}
\end{array}\right]^{\mathrm{T}} \quad \text { and } \quad \delta:=\left[\begin{array}{c}
\delta^{\prime \prime} \\
\delta^{\prime}
\end{array}\right] .
$$

For $a$ and $b$ in $\left(\frac{1}{2} \boldsymbol{Z}\right)^{g}$, we let

$$
\begin{aligned}
\vartheta\left[\begin{array}{l}
a \\
b
\end{array}\right](z) & =\vartheta\left[\begin{array}{l}
a \\
b
\end{array}\right](z ; Z) \\
& =\sum_{n \in \boldsymbol{Z}^{g}} \exp \left[2 \pi \mathrm{i}\left\{\frac{1}{2}(n+a)^{\mathrm{T}} Z(n+a)+(n+a)^{\mathrm{T}}(z+b)\right\}\right] .
\end{aligned}
$$

Then the hyperelliptic sigma function on $\boldsymbol{C}^{g}$ associated with $C$ is defined by

$$
\tilde{\sigma}(u)=\exp \left(-\frac{1}{2} u \eta^{\prime} \omega^{\prime-1} u^{\mathrm{T}}\right) \vartheta[\delta]\left(\omega^{\prime-1} u^{\mathrm{T}} ; Z\right)
$$

up to a multiplicative constant, where $u=\left(u_{1}, u_{2}, \ldots, u_{g}\right)$. We shall fix this constant later.

Definition 4.1. We define the Sato weight by taking the weight of $u_{j}$ to be $2(g-j)+1$. When we consider several variables $u^{(1)}, \ldots, u^{(n)}$ on $\kappa^{-1} \iota(C)$ for some $n$, we also regard the Sato weight of each $u_{j}^{(i)}$ as being $2(g-j)+1$. Moreover, we define the Sato weight of $\lambda_{j}$ to be $-2 j$.

To fix the multiplicative constant above, we recall the following.

\section{Lemma 4.2.}

(i) The power-series expansion of $\tilde{\sigma}(u)$ with respect to $u_{1}, u_{2}, \ldots, u_{g}$ has polynomial coefficients in $\lambda_{0}, \lambda_{1}, \ldots, \lambda_{2 g+1}$, and is homogeneous in the Sato weight.

(ii) The terms of least total degree of the power-series expansion of the function $\tilde{\sigma}(u)$ with respect to the variables $u_{1}, \ldots, u_{g}$ is either a non-zero constant multiple of the Hankel-type determinant

$$
\left|\begin{array}{cccc}
u_{1} & u_{2} & \cdots & u_{(g+1) / 2} \\
u_{2} & u_{3} & \cdots & u_{(g+3) / 2} \\
\vdots & \vdots & \ddots & \vdots \\
u_{(g+1) / 2} & u_{(g+3) / 2} & \cdots & u_{g}
\end{array}\right|
$$


if $g$ is odd, or of

if $g$ is even.

$$
\left|\begin{array}{cccc}
u_{1} & u_{2} & \cdots & u_{g / 2} \\
u_{2} & u_{3} & \cdots & u_{(g+2) / 2} \\
\vdots & \vdots & \ddots & \vdots \\
u_{g / 2} & u_{(g+2) / 2} & \cdots & u_{g-1}
\end{array}\right|
$$

Proof. While statement (i) is shown in the proof of Corollary 1 of [7], it can also be proved as follows. This proof was supplied by the referee. For any non-zero constant $\alpha$, the transform given by $x \mapsto \alpha^{2} x, y \mapsto \alpha^{2 g+1} y, \lambda_{j} \mapsto \alpha^{2 j} \lambda_{j}$ defines an isomorphism of the curve $C$ that maps $x^{j} \mathrm{~d} x / 2 y \mapsto \alpha^{2(j-g)-1} x^{j} \mathrm{~d} x / 2 y$, and hence $u_{j} \mapsto \alpha^{2(j-g)-1} u_{j}$. Since the position of the zeros and the periodicity of $\sigma(u)$ are invariant under this transform, and $\sigma(u)$ is determined up to a constant multiple by its zeros and periodicity, its expansion should be homogeneous with respect to the Sato weight. Statement (ii) is proved in Proposition 2.2 in [5, p. 32] or in [2, pp. 359 and 360].

In this paper we let $\sigma(u)$ be the function such that it is a constant multiple of $\tilde{\sigma}(u)$ and the terms of least total degree of its power-series expansion at $u=(0,0, \ldots, 0)$ are just the Hankel-type determinant above.

For $u \in \boldsymbol{C}^{g}$ we conventionally denote by $u^{\prime}$ and $u^{\prime \prime}$ the elements of $\boldsymbol{R}^{g}$ such that $u=\omega^{\prime} u^{\prime}+\omega^{\prime \prime} u^{\prime \prime}$, where $\left[\omega^{\prime} \omega^{\prime \prime}\right]$ are the period matrices above. We define a $\boldsymbol{C}$-valued $\boldsymbol{R}$-bilinear form $L(\cdot, \cdot)$ by

$$
L(u, v)=u\left(\eta^{\prime} v^{\prime}+\eta^{\prime \prime} v^{\prime \prime}\right)^{\mathrm{T}}
$$

for $u, v \in \boldsymbol{C}^{g}$. For $\ell$ in $\Lambda$, the lattice of periods as defined in $\S 2$, let

$$
\chi(\ell)=\exp \left[2 \pi \mathrm{i}\left(\ell^{\prime} \delta^{\prime \prime}-\ell^{\prime \prime} \delta^{\prime}\right)-\pi \mathrm{i} \ell^{\prime} \ell^{\prime \prime}\right] .
$$

Lemma 4.3. The function $\sigma(u)$ is an odd function if $g \equiv 1$ or 2 modulo 4 , and an even function if $g \equiv 3$ or 0 modulo 4 .

Proof. See [17, p. 3.97 and p. 3.100].

Lemma 4.4 (the translational relation). The function $\sigma(u)$ satisfies

$$
\sigma(u+\ell)=\chi(\ell) \sigma(u) \exp L\left(u+\frac{1}{2} \ell, \ell\right)
$$

for all $u \in \boldsymbol{C}^{g}$ and $\ell \in \Lambda$.

For a proof of this formula we refer the reader to [3, p. 286].

Remark 4.5. The Riemann form of $\sigma(u)$, which is defined by $E(u, v)=L(u, v)-$ $L(v, u)\left(u, v \in \boldsymbol{C}^{g}\right)$ is simply written as $E(u, v)=2 \pi \mathrm{i}\left(u^{\prime} v^{\prime \prime^{\mathrm{T}}}-u^{\prime \prime} v^{\prime^{\mathrm{T}}}\right)$ (see Lemma 3.1.2(2) in $[\mathbf{1 8}$, p. 396]). Hence, $E(\cdot, \cdot)$ is an i $\boldsymbol{R}$-valued form and $2 \pi \mathrm{i} \boldsymbol{Z}$-valued on $\Lambda \times \Lambda$. In particular, the pfaffian of $E(\cdot, \cdot)$ is 1 . This simple expression is one of the convenient properties for distinguishing $\sigma(u)$ from the theta series without multiplication by an exponential pre-factor in (4.1). 
Let $J$ be the Jacobian variety of the curve $C$. We identify $J$ with the Picard group $\operatorname{Pic}^{\circ}(C)$ of the linearly equivalent classes of divisors of degree zero of $C$. Let $\operatorname{Sym}^{g}(C)$ be the $g$ th symmetric product of $C$. Then we have a birational map

$$
\begin{aligned}
\operatorname{Sym}^{g}(C) & \rightarrow \operatorname{Pic}^{\circ}(C)=J, \\
\left(P_{1}, \ldots, P_{g}\right) & \mapsto \text { the class of } P_{1}+\cdots+P_{g}-g \cdot \infty .
\end{aligned}
$$

As an analytic manifold, $J$ is identified with $\boldsymbol{C}^{g} / \Lambda$. We denote by $\kappa$ the canonical map $\boldsymbol{C}^{g} \rightarrow \boldsymbol{C}^{g} / \Lambda=J$. We embed $C$ into $J$ by $\iota: Q \mapsto Q-\infty$. For each $n=1, \ldots, g-1$ let $\Theta^{[n]}$ be the subvariety of $J$ determined by the set of classes of the form $P_{1}+\cdots+P_{n}-n \cdot \infty$. This is called the standard theta subvariety of dimension $n$. Obviously, $\Lambda=\kappa^{-1} \iota(\infty)$ and $\Theta^{[1]}=\iota(C)$.

Analytically, each point $\left(P_{1}, \ldots, P_{g}\right)$ of $\operatorname{Sym}^{g}(C)$ is canonically mapped to

$$
u=\left(u_{1}, \ldots, u_{g}\right)=\left(\int_{\infty}^{P_{1}}+\cdots+\int_{\infty}^{P_{g}}\right)\left(\omega_{1}, \ldots, \omega_{g}\right),
$$

and $\sigma(u)$ is regarded as a function on the universal covering space $\boldsymbol{C}^{g}$ of $J$ with the canonical map $\kappa$ above and the natural coordinate $u$ of $\boldsymbol{C}^{g}$.

If $u=\left(u_{1}, \ldots, u_{g}\right)$ is in $\kappa^{-1} \iota(C)$, we denote by $(x(u), y(u))$ the coordinate of the point on $C$ corresponding to $u$, so that

$$
u=\int_{\infty}^{(x(u), y(u))}\left(\frac{1}{2 y}, \frac{x}{2 y}, \ldots, \frac{x^{g-1}}{2 y}\right) \mathrm{d} x
$$

with appropriate choice for a path of the integrals. Then we have that $x(-u)=x(u)$, $y(-u)=-y(u)$ and $(x(0,0, \ldots, 0), y(0,0, \ldots, 0))=\infty$. We frequently use the following lemma in the rest of the paper.

Lemma 4.6. Suppose $u \in \kappa^{-1} \iota(C)$. The Laurent expansions of $x(u)$ and $y(u)$ at $u=(0, \ldots, 0)$ on the pull-back $\kappa^{-1} \iota(C)$ of $C$ to $\boldsymbol{C}^{g}$ are

$$
x(u)=\frac{1}{u_{g}{ }^{2}}+\left(d^{\circ}\left(u_{g}\right) \geqslant 0\right), \quad y(u)=-\frac{1}{u_{g}^{2 g+1}}+\left(d^{\circ}\left(u_{g}\right) \geqslant-2 g+1\right)
$$

with their coefficients in $\boldsymbol{Q}\left[\lambda_{0}, \lambda_{1}, \ldots, \lambda_{2 g+1}\right]$. Moreover, $x(u)$ and $y(u)$ are homogeneous in the Sato weight -2 and $-(2 g+1)$, respectively.

Proof. We take $t=1 / \sqrt{x}$ as a local parameter at $\infty$ along $\iota(C)$. If $u$ is in $\kappa^{-1} \iota(C)$ and sufficiently near $(0,0, \ldots, 0)$, we can assume that $t, u=\left(u_{1}, \ldots, u_{g}\right)$ and $(x, y)$ are coordinates of the same point on $C$. Then

$$
\begin{aligned}
u_{g} & =\int_{\infty}^{(x, y)} \frac{x^{g-1} \mathrm{~d} x}{2 y} \\
& =\int_{\infty}^{(x, y)} \frac{x^{-3 / 2} \mathrm{~d} x}{2 \sqrt{1+\lambda_{1} / x+\cdots+\lambda_{2 g+1} / x^{2 g+1}}} \\
& =\int_{0}^{t} \frac{t^{3}\left(-2 / t^{3}\right) \mathrm{d} t}{2+\left(d^{\circ} \geqslant 1\right)} \\
& =-t+\left(d^{\circ}(t) \geqslant 2\right) .
\end{aligned}
$$


Hence $x(u)=1 / u_{g}^{2}+\left(d^{\circ}\left(u_{g}\right) \geqslant-1\right)$ and our assertion is proved, because $x(-u)=x(u)$ and $y(-u)=-y(u)$. The rest of the statements are obvious from the calculation above.

Lemma 4.7. If $u=\left(u_{1}, u_{2}, \ldots, u_{g}\right)$ is a variable on $\kappa^{-1}\left(\Theta^{[1]}\right)$, then

$$
\begin{aligned}
u_{1} & =\frac{1}{2 g-1} u_{g}^{2 g-1}+\left(d^{\circ}\left(u_{g}\right) \geqslant 2 g\right), \\
u_{2} & =\frac{1}{2 g-3} u_{g}^{2 g-3}+\left(d^{\circ}\left(u_{g}\right) \geqslant 2 g-2\right), \\
& \vdots \\
u_{g-1} & =\frac{1}{3} u_{g}{ }^{3}+\left(d^{\circ}\left(u_{g}\right) \geqslant 4\right)
\end{aligned}
$$

with the coefficients in $\boldsymbol{Q}\left[\lambda_{0}, \lambda_{1}, \ldots, \lambda_{2 g+1}\right]$, and these expansions are homogeneous with respect to the Sato weight.

Proof. The assertions are easily obtained by similar calculations to those used in the proof of Lemma 4.6 .

Remark 4.8. The second set of equalities in (2.2) is the canonical limit of the equalities in Lemma 4.7 when we let all the coefficients $\lambda_{1}, \ldots, \lambda_{2 g+1}$ tend to 0 , because of the homogeneity there.

\section{The Schur-Weierstrass polynomial and the sigma function}

The polynomial $S(u)$ is closely related to the function $\sigma(u)$ as follows. We put

$$
S_{i_{1} i_{2} \cdots i_{n}}(u)=\frac{\partial}{\partial u_{i_{1}}} \frac{\partial}{\partial u_{i_{2}}} \cdots \frac{\partial}{\partial u_{i_{n}}} S(u), \quad \sigma_{i_{1} i_{2} \cdots i_{n}}(u)=\frac{\partial}{\partial u_{i_{1}}} \frac{\partial}{\partial u_{i_{2}}} \cdots \frac{\partial}{\partial u_{i_{n}}} \sigma(u) .
$$

Proposition 5.1. The function $\sigma(u)$ has the power-series expansion

$$
\sigma(u)=(-1)^{g(g-1)(g-3) / 2} S(u)+\left(d^{\circ}\left(\lambda_{1}, \lambda_{2}, \ldots, \lambda_{2 g+1}\right) \geqslant 1\right) \in \boldsymbol{Q}\left[\lambda_{1}, \ldots, \lambda\right]\left[\left[u_{1}, \ldots, u_{g}\right]\right]
$$

at $u=(0,0, \ldots, 0)$, and is homogeneous with respect to the Sato weight.

Proof. It is easy to see from the proof of the Corollary 1 of [7] that the power-series expansion of $\sigma(u)$ with respect to $u_{1}, u_{2}, \ldots, u_{g}$ belongs to the formal power-series ring $\boldsymbol{Q}\left[\lambda_{0}, \lambda_{1}, \ldots, \lambda_{2 g+1}\right] \times\left[\left[u_{1}, u_{2}, \ldots, u_{g}\right]\right]$, namely, is expanded over the rational numbers. 
If $g$ is odd, then

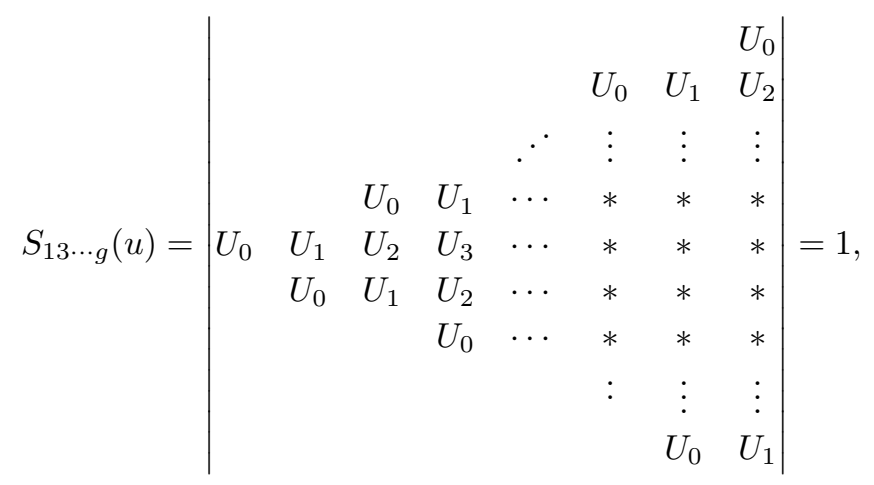

and $\sigma_{13 \cdots g}(0,0, \ldots, 0)=1$ by Lemma 4.2 . If $g$ is even, then

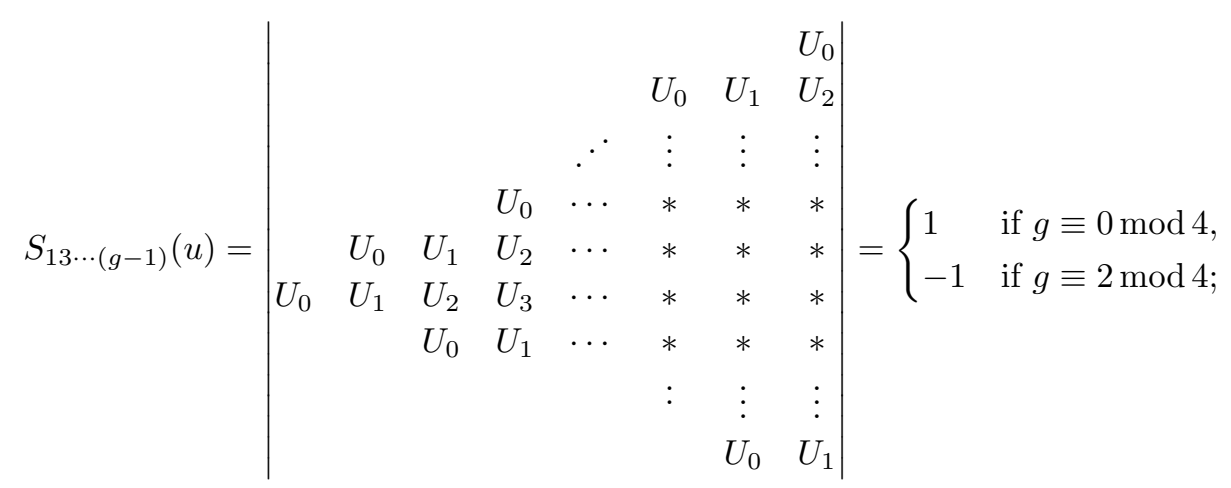

and $\sigma_{13 \cdots(g-1)}(0,0, \ldots, 0)=1$ by Lemma 4.2 again. Hence the form of expansion of $\sigma(u)$ follows straight from Theorem 6.3 of $[\mathbf{6}]$. The last statement is a repetition of Lemma 4.2(i).

Corollary 5.2. The derivatives of $\sigma(u)$ and $S(u)$ are related as follows:

$$
\sigma_{i_{1} i_{2} \cdots i_{n}}(u)=(-1)^{g(g-1)(g-3) / 2} S_{i_{1} i_{2} \cdots i_{n}}(u)+\left(d^{\circ}\left(\lambda_{1}, \lambda_{2}, \ldots, \lambda_{2 g+1}\right) \geqslant 1\right) .
$$

This series is homogeneous in the Sato weight.

Proof. This is obvious from Proposition 5.1.

\section{The vanishing structure of the sigma function and of its derivatives}

We investigate vanishing structure of $\sigma(u)$ and of its derivatives by using the Riemann singularity theorem and calculations using the Brill-Noether matrices. The following is fundamental for us. 
Proposition 6.1 (Riemann singularity theorem). For a given $u \in \kappa^{-1}\left(\Theta^{[g-1]}\right)$, we denote a divisor on the curve $C$ which corresponds the point $u$ modulo $\Lambda$ by $P_{1}+$ $\cdots+P_{g-1}-(g-1) \cdot \infty$. Then

$$
\operatorname{dim} \Gamma\left(C, \mathcal{O}\left(P_{1}+\cdots+P_{g-1}\right)\right)=r+1
$$

if and only if both of the following hold:

(i) $\sigma_{i_{1} i_{2} \cdots i_{h}}(u)=0$ for any $h \leqslant r$ and for any $i_{1}, \ldots, i_{h} \in\{1,2, \ldots, g\}$;

(ii) there exists an $(r+1)$-tuple $\left\{i_{1}, i_{2}, \ldots, i_{r+1}\right\}$ such that $\sigma_{i_{1} i_{2} \cdots i_{r+1}}(u) \neq 0$.

Proof. By (4.1) we easily restate the fact stated in [1, pp. 226 and 227] into the statements above.

To compute the dimension of the zeroth cohomology group above, we recall the BrillNoether matrix, defined as follows. We fix a local parameter of every point of $C$. To clarify the following argument we define the local parameter $t$ at each point $P$ by

$$
t= \begin{cases}y & \text { if } y(P)=0 \\ x-x(P) & \text { if } y(P) \neq 0 \text { and } P \neq \infty \\ \frac{1}{\sqrt{x}} & \text { if } P=\infty\end{cases}
$$

We denote by $\Omega^{1}$ the sheaf of differential forms of the first kind. For a point $P$ of $C$, let $t$ be the local parameter defined above. We denote by $P_{t}$ the point of $C$ at which the value of the local parameter is $t$. Then we define for $\mu \in \Gamma\left(C, \Omega^{1}\right)$

$$
\delta^{\ell} \mu(P)=\left.\frac{\mathrm{d}^{\ell}}{\mathrm{d} t^{\ell}} \int_{\infty}^{P_{t}} \mu\right|_{t=0} .
$$

Since $\mu$ is a holomorphic form, $\delta^{\ell} \mu(P)$ takes a finite value at every point $P$. Let $D:=\sum_{j=1}^{k} n_{j} P_{j}$ with different values of $P_{j}$ be an effective divisor. A matrix with $\operatorname{deg} D:=\sum n_{j}$ rows and $g$ columns is called the Brill-Noether matrix for $D$ if its $\left(n_{1}+\cdots+n_{j-1}+\ell, i\right)$-entry is $\delta^{\ell} \omega_{i}\left(P_{j}\right)$, where $1 \leqslant \ell \leqslant n_{j}$ and $\omega_{i}=\left(x^{i-1} / 2 y\right) \mathrm{d} x$. We denote by $B(D)$ the Brill-Noether matrix for $D$. Our computation starts with the following.

Proposition 6.2. Let $D$ be an effective divisor of $C$. Then

$$
\operatorname{dim} \Gamma(C, \mathcal{O}(D))=\operatorname{deg} D+1-\operatorname{rank} B(D) .
$$

Proof. For $\mu \in \Gamma\left(C, \Omega^{1}\right)$, we can find uniquely the set of elements $c_{1}, \ldots, c_{g} \in \boldsymbol{C}$ such that $\mu=c_{1} \omega_{1}+\cdots+c_{g} \omega_{g}$. Let $D=\sum_{j=1}^{k} n_{j} P_{j}$. In this situation, the three statements

(i) $\mu \in \Gamma\left(C, \Omega^{1}(-D)\right)$,

(ii) $\delta^{\ell} \mu\left(P_{j}\right)=0$ for all $j$ and $\ell$ with $1 \leqslant j \leqslant k$ and $1 \leqslant \ell \leqslant n_{j}$, and 
(iii) $B(D)\left[\begin{array}{c}c_{1} \\ \vdots \\ c_{g}\end{array}\right]=\left[\begin{array}{c}0 \\ \vdots \\ 0\end{array}\right]$

are equivalent. So $\operatorname{dim} \Gamma\left(C, \Omega^{1}(-D)\right)=g-\operatorname{rank} B(D)$. The Riemann-Roch theorem states

$$
\operatorname{dim} \Gamma(C, \mathcal{O}(D))=\operatorname{deg} D-g+1+\operatorname{dim} \Gamma\left(C, \Omega^{1}(-D)\right) .
$$

Hence $\operatorname{dim} \Gamma(C, \mathcal{O}(D))=\operatorname{deg} D+1-\operatorname{rank} B(D)$.

To compute the rank of $B(D)$ we need only consider the case where $D$ is of the form $D=P_{1}+P_{2}+\cdots+P_{n}+(g-n-1) \cdot \infty \quad\left(P_{i} \neq P_{j}, \bar{P}_{j}\right.$ for any $i \neq j$, and $\left.P_{j} \neq \infty\right)$, where $\bar{P}_{j}$ is the hyperelliptic involution of $P_{j}$. Then the matrix $B(D)$ is given by

$$
\left[\begin{array}{cccc|ccccc}
\frac{1}{2 y}\left(P_{1}\right) & \frac{x}{2 y}\left(P_{1}\right) & \ldots & \frac{x^{n-1}}{2 y}\left(P_{1}\right) & \frac{x^{n}}{2 y}\left(P_{1}\right) & \ldots & \frac{x^{g-3}}{2 y}\left(P_{1}\right) & \frac{x^{g-2}}{2 y}\left(P_{1}\right) & \frac{x^{g-1}}{2 y}\left(P_{1}\right) \\
\vdots & \vdots & \ddots & \vdots & \vdots & \ddots & \vdots & \vdots & \vdots \\
\frac{1}{2 y}\left(P_{n}\right) & \frac{x}{2 y}\left(P_{n}\right) & \ldots & \frac{x^{n-1}}{2 y}\left(P_{n}\right) & \frac{x^{n}}{2 y}\left(P_{n}\right) & \ldots & \frac{x^{g-3}}{2 y}\left(P_{n}\right) & \frac{x^{g-2}}{2 y}\left(P_{n}\right) & \frac{x^{g-1}}{2 y}\left(P_{n}\right) \\
\hline & & & 0 & \cdots & 0 & 0 & 1 \\
& & & 0 & \cdots & 0 & 0 & \\
& & & & & \cdots & 0 & 1 & \\
& & & & \cdots & 0 & & \\
& & & & \cdots & 1 & &
\end{array}\right] .
$$

Here the right lower block was calculated in a similar way to Lemmas 4.6 and 4.7 . Therefore, the rank of $B(D)$ is $n+(g-n-1) / 2$ or $n+(g-n) / 2$ according to whether $g-n$ is odd or even. Combining up the considerations above, we have

$$
\begin{aligned}
\operatorname{dim} \Gamma\left(C, \mathcal{O}\left(P_{1}+P_{2}+\cdots+P_{n}+(g-n-1) \infty\right)\right) & =g-1-(n+\lfloor(g-n) / 2\rfloor) \\
& =\lfloor(g-n-1) / 2\rfloor+1 .
\end{aligned}
$$

Again we denote by $u$ the point in $\kappa^{-1}\left(\Theta^{[g-1]}\right)$ corresponding to $P_{1}+P_{2}+\cdots+P_{n}+(g-$ $n-1) \infty-(g-1) \infty$. Proposition 6.1 yields that if $h \leqslant\lfloor(g-n-1) / 2\rfloor$, then $\sigma_{i_{1} i_{2} \cdots i_{h}}(u)=0$ for all $i_{1}, \ldots, i_{h}$ and $\sigma_{j_{1} j_{2} \cdots j_{\lfloor(g-n-1) / 2\rfloor+1}}(u) \neq 0$ for some $j_{1}, j_{2}, \ldots, j_{\lfloor(g-n-1) / 2\rfloor+1}$. Here we record the first fact as follows.

Lemma 6.3. We fix the genus $g$. Let $n(\leqslant g-1)$ and $h$ be integers such that $0 \leqslant$ $h \leqslant\lfloor(g-n-1) / 2\rfloor$. Let $i_{1}, i_{2}, \ldots, i_{h}$ be arbitrary $h$ elements in $\{1,2, \ldots, g\}$. Then the function $u \mapsto \sigma_{i_{1} i_{2} \cdots i_{h}}(u)$ on $\kappa^{-1}\left(\Theta^{[n]}\right)$ is identically zero. 
Table 1. Table of $\sigma_{\natural^{n}}(u)$

\begin{tabular}{|c|c|c|c|c|c|c|c|c|c|c|c|}
\hline genus & $\sigma_{\sharp}$ & $\sigma_{b}$ & $\sigma_{\natural^{3}}$ & $\sigma_{\natural^{4}}$ & $\sigma_{\natural^{5}}$ & $\sigma_{\natural^{6}}$ & $\sigma_{\natural^{7}}$ & $\sigma_{\natural^{8}}$ & $\sigma_{\natural^{9}}$ & $\sigma_{\natural^{10}}$ & $\ldots$ \\
\hline 1 & $\sigma$ & $\sigma$ & $\sigma$ & $\sigma$ & $\sigma$ & $\sigma$ & $\sigma$ & $\sigma$ & $\sigma$ & $\sigma$ & $\ldots$ \\
\hline 2 & $\sigma_{2}$ & $\sigma$ & $\sigma$ & $\sigma$ & $\sigma$ & $\sigma$ & $\sigma$ & $\sigma$ & $\sigma$ & $\sigma$ & $\ldots$ \\
\hline 3 & $\sigma_{2}$ & $\sigma_{3}$ & $\sigma$ & $\sigma$ & $\sigma$ & $\sigma$ & $\sigma$ & $\sigma$ & $\sigma$ & $\sigma$ & $\ldots$ \\
\hline 4 & $\sigma_{24}$ & $\sigma_{3}$ & $\sigma_{4}$ & $\sigma$ & $\sigma$ & $\sigma$ & $\sigma$ & $\sigma$ & $\sigma$ & $\sigma$ & $\ldots$ \\
\hline 5 & $\sigma_{24}$ & $\sigma_{35}$ & $\sigma_{4}$ & $\sigma_{5}$ & $\sigma$ & $\sigma$ & $\sigma$ & $\sigma$ & $\sigma$ & $\sigma$ & $\ldots$ \\
\hline 6 & $\sigma_{246}$ & $\sigma_{35}$ & $\sigma_{46}$ & $\sigma_{5}$ & $\sigma_{6}$ & $\sigma$ & $\sigma$ & $\sigma$ & $\sigma$ & $\sigma$ & $\ldots$ \\
\hline 7 & $\sigma_{246}$ & $\sigma_{357}$ & $\sigma_{46}$ & $\sigma_{57}$ & $\sigma_{6}$ & $\sigma_{7}$ & $\sigma$ & $\sigma$ & $\sigma$ & $\sigma$ & $\ldots$ \\
\hline 8 & $\sigma_{2468}$ & $\sigma_{357}$ & $\sigma_{468}$ & $\sigma_{57}$ & $\sigma_{68}$ & $\sigma_{7}$ & $\sigma_{8}$ & $\sigma$ & $\sigma$ & $\sigma$ & $\ldots$ \\
\hline 9 & $\sigma_{2468}$ & $\sigma_{3579}$ & $\sigma_{468}$ & $\sigma_{579}$ & $\sigma_{68}$ & $\sigma_{79}$ & $\sigma_{8}$ & $\sigma_{9}$ & $\sigma$ & $\sigma$ & $\ldots$ \\
\hline$\vdots$ & $\vdots$ & $\vdots$ & $\vdots$ & $\vdots$ & $\vdots$ & $\vdots$ & $\vdots$ & $\vdots$ & $\vdots$ & $\vdots$ & $\ddots$ \\
\hline
\end{tabular}

\section{Special derivatives of the sigma function}

We will introduce some special derivatives of the sigma function. These are important to state our Frobenius-Stickelberger-type formula.

Definition 7.1. Let $\natural^{n}$ be the set defined in Definition 3.1. Then we define a derivative $\sigma_{\natural^{n}}(u)$ of $\sigma(u)$ by

$$
\sigma_{\mathfrak{\natural}^{n}}(u)=\left(\prod_{i \in \mathfrak{\natural}^{n}} \frac{\partial}{\partial u_{i}}\right) \sigma(u) .
$$

In particular, we define

$$
\sigma_{\sharp}(u)=\sigma_{\natural^{1}}(u), \quad \sigma_{b}(u)=\sigma_{\natural^{2}}(u) .
$$

These functions are given in Table 1.

We now prepare tools for investigating the zeros of these derivatives.

Lemma 7.2. Suppose $0 \leqslant n \leqslant g-1$.

Let $\check{\natural}^{n}$ be a proper subset of $\natural^{n}$, and let

$$
\sigma_{\check{\natural ̆}^{n}}(u)=\left(\prod_{i \in \check{\natural}^{n}} \frac{\partial}{\partial u_{i}}\right) \sigma(u) .
$$

Then the function $u \mapsto \sigma_{\check{\natural}^{n}}(u)$ on $\kappa^{-1}\left(\Theta^{[n]}\right)$ is identically zero.

Proof. Since the number of elements in the set $\natural^{n}$ is $\lfloor(g-n+1) / 2\rfloor$, we see the number of elements in $\check{\natural}^{n}$ is less than or equal to $\lfloor(g-n-1) / 2\rfloor$. Hence we have the assertion from Lemma 6.3.

Lemma 7.3 (the translational relation). Let $n$ be an integer such that $1 \leqslant n \leqslant$ $g-1$. Assume $u$ belongs to $\kappa^{-1}\left(\Theta^{[n]}\right)$. Then we have

$$
\sigma_{\natural^{n}}(u+\ell)=\chi(\ell) \sigma_{\natural^{n}}(u) \exp L\left(u+\frac{1}{2} \ell, \ell\right)
$$

for all $\ell \in \Lambda$. 
Proof. This follows from Lemma 7.2 using Lemma 4.4 and Definition 7.1.

Proposition 7.4. Let $n$ be an integer such that $1 \leqslant n \leqslant g-1$. The space spanned by the functions $u \mapsto \varphi(u)$ on $\kappa^{-1}\left(\Theta^{[n]}\right)$ vanishing only on $\kappa^{-1}\left(\Theta^{[n-1]}\right)$ and satisfying the equation

$$
\varphi(u+\ell)=\chi(\ell) \varphi(u) \exp L\left(u+\frac{1}{2} \ell, \ell\right)
$$

for all $\ell \in \Lambda$ is one dimensional.

Proof. Let $\varphi_{1}(u)$ and $\varphi_{2}(u)$ be non-trivial functions on $\kappa^{-1}\left(\Theta^{[n]}\right)$ with the stated properties. Since $\Theta^{[n-1]}$ is a prime divisor of the variety $\Theta^{[n]}$, the equations assumed for these functions imply that we may assume that the vanishing order of $\varphi_{2}(u)$ on $\kappa^{-1}\left(\Theta^{[n-1]}\right)$ is less than or equal to that of $\varphi_{1}(u)$. Then the function $\varphi_{1} / \varphi_{2}$ is holomorphic on $\kappa^{-1}\left(\Theta^{[n]}\right)$. Classically, this is a situation where we might use a special case of Hartogs's analytic continuation theorem. On the other hand, we have

$$
\frac{\varphi_{1}}{\varphi_{2}}(u+\ell)=\frac{\varphi_{1}}{\varphi_{2}}(u) \text { for all } u \in \kappa^{-1}\left(\Theta^{[n]}\right) \text { and } \ell \in \Lambda,
$$

by the supposed equations. Therefore, $\varphi_{1} / \varphi_{2}$ can be regarded as a holomorphic function on $\Theta^{[n]}$. Hence this is a constant function, by Liouville's theorem.

Proposition 7.5. Let $v$ be a variable on $\kappa^{-1}\left(\Theta^{[1]}\right)$. Then we have the following.

(i) The function $v \mapsto \sigma_{\sharp}(v)$ has a zero of order $g$ at $v=(0,0, \ldots, 0)$ modulo $\Lambda$ and no zero elsewhere. This function has an expansion of the form

$$
\sigma_{\sharp}(v)=(-1)^{(g-2)(g-3) / 2} v_{g}^{g}+\left(d^{\circ}\left(v_{g}\right) \geqslant g+2\right) .
$$

(ii) Let $n$ be an integer such that $1 \leqslant n \leqslant g-1$. Suppose $v, u^{(1)}, u^{(2)}, \ldots, u^{(n)}$ belong to $\kappa^{-1}\left(\Theta^{[1]}\right)$. If $u^{(1)}+\cdots+u^{(n)} \notin \kappa^{-1}\left(\Theta^{[n-1]}\right)$, then the function $v \mapsto$ $\sigma_{\mathfrak{\natural}^{n+1}}\left(u^{(1)}+\cdots+u^{(n)}+v\right)$ has zeros of order 1 at $v=-u^{(1)}, \ldots,-u^{(n)}$, a zero of order $g-n$ at $v=(0,0, \ldots, 0)$ modulo $\Lambda$ and no other zero elsewhere. This function has an expansion of the form

$$
\begin{aligned}
\sigma_{\natural^{n+1}}\left(u^{(1)}\right. & \left.+\cdots+u^{(n)}+v\right) \\
& =(-1)^{(g-n)(g-n-1) / 2} \sigma_{\natural^{n}}\left(u^{(1)}+\cdots+u^{(n)}\right) v_{g}{ }^{g-n}+\left(d^{\circ}\left(v_{g}\right) \geqslant g-n+1\right) .
\end{aligned}
$$

(iii) If $u \notin \kappa^{-1}\left(\Theta^{[g-1]}\right)$, then $\sigma(u) \neq 0$.

Proof. Statement (iii) is well known (see Theorem 5.3 in $[\mathbf{1 9}$, p. 3.80]). We prove (i) and (ii). The usual argument by integration of the logarithm of

$$
\begin{aligned}
\sigma_{\mathfrak{\natural}^{n+1}}\left(u^{(1)}\right. & \left.+\cdots+u^{(n)}+v+\ell\right) \\
& =\chi(\ell) \sigma_{\natural^{n+1}}\left(u^{(1)}+\cdots+u^{(n)}+v\right) \exp L\left(u^{(1)}+\cdots+u^{(n)}+v+\frac{1}{2} \ell, \ell\right)
\end{aligned}
$$


of Lemma 7.3 along the boundary of a polygon representation of the Riemann surface of $C$ shows either that the functions $v \mapsto \sigma_{\sharp}(v)$ and $v \mapsto \sigma_{\natural^{n+1}}\left(u^{(1)}+\cdots+u^{(n)}+v\right)$ above have exactly $g$ zeros modulo $\Lambda$ or that they vanish identically (see [13, p. 147] for details). These functions, however, do not vanish identically because of Proposition 3.4 and Corollary 5.2. The other statements of (i) follow from Corollary 5.2 and Proposition 3.4(i).

We prove the rest of statement (ii). Since the number of elements in $\natural^{n+1}$ is $\lfloor(g-n) / 2\rfloor$, Lemma 6.3 shows that the function

$$
v \mapsto \sigma_{\natural^{n+1}}\left(u^{(1)}+\cdots+u^{(n)}+v\right)
$$

has zeros at least at $v=-u^{(1)}, \ldots,-u^{(n)}$. For $v \in \kappa^{-1}\left(\Theta^{[1]}\right)$, we let

$$
\sigma_{\mathfrak{\natural}^{n+1}}\left(u^{(1)}+\cdots+u^{(n)}+v\right)=\sum_{j=0}^{\infty} \varphi^{(j)}\left(u^{(1)}+\cdots+u^{(n)}\right) v_{g}^{j},
$$

where $\varphi^{(j)}$ are certain functions on $\kappa^{-1}\left(\Theta^{[n]}\right)$. Let the Sato weight of $\sigma_{\mathfrak{t}^{n+1}}\left(u^{(1)}+\cdots+\right.$ $\left.u^{(n)}+v\right)$ be $m$, so that $m=n g-\frac{1}{2} n(n+1)$. Let $j_{0}$ be the minimal $j$ s such that $\varphi^{(j)}\left(u^{(1)}+\cdots+u^{(n)}\right)$ is not identically 0 as a function of the variables $u^{(1)}, \ldots, u^{(n)}$. So, we can write

$$
\sigma_{\natural^{n+1}}\left(u^{(1)}+\cdots+u^{(n)}+v\right)=\varphi^{\left(j_{0}\right)}\left(u^{(1)}+\cdots+u^{(n)}\right) v_{g}^{j_{0}}+\cdots .
$$

Then the Sato weight of $\varphi^{\left(j_{0}\right)}\left(u^{(1)}+\cdots+u^{(n)}\right)$ is $m-j_{0}$.

We claim that the function $u \mapsto \varphi^{\left(j_{0}\right)}(u)$ on $\kappa^{-1}\left(\Theta^{[n]}\right)$ satisfies the equation in Proposition 7.4. To prove it, let us take $\ell \in \Lambda$ and $u \in \kappa^{-1}\left(\Theta^{[n]}\right)$. Then, since $u+\ell \in \kappa^{-1}\left(\Theta^{[n]}\right)$, we have

$$
\sigma_{\mathrm{\natural}^{n+1}}(u+v+\ell)=\varphi^{\left(j_{0}\right)}(u+\ell) v_{g}^{j_{0}}+\cdots .
$$

On the other hand, the translational relation for $\sigma_{\mathfrak{\natural}^{n+1}}$ gives

$$
\begin{aligned}
\sigma_{\natural^{n+1}}(u+v+\ell) & =\chi(\ell) \sigma_{\mathfrak{\natural}^{n+1}}(u+v) \exp L\left(u+v+\frac{1}{2} \ell, \ell\right) \\
& =\chi(\ell)\left(\varphi^{\left(j_{0}\right)}(u) v_{g}{ }^{j_{0}}+\cdots\right) \exp L\left(u+v+\frac{1}{2} \ell, \ell\right) \\
& =\chi(\ell)\left(\varphi^{\left(j_{0}\right)}(u) v_{g}{ }^{j_{0}}+\cdots\right)\left\{\exp L\left(u+\frac{1}{2} \ell, \ell\right)+\left(d^{\circ}\left(v_{g}\right) \geqslant 1\right)\right\} \\
& =\chi(\ell) \varphi^{\left(j_{0}\right)}(u) \exp L\left(u+\frac{1}{2} \ell, \ell\right) v_{g}{ }^{j_{0}}+\left(d^{\circ}\left(v_{g}\right) \geqslant\left(j_{0}+1\right)\right) .
\end{aligned}
$$

Comparing these two equations, we see that

$$
\varphi^{\left(j_{0}\right)}(u+\ell)=\chi(\ell) \varphi^{\left(j_{0}\right)}(u) \exp L\left(u+\frac{1}{2} \ell, \ell\right) .
$$

This proves the claim.

Proposition 7.4 yields that $\varphi^{\left(j_{0}\right)}(u)$ is, as a function on $\kappa^{-1}\left(\Theta^{[n]}\right)$, equal to $\sigma_{\natural^{n}}(u)$ up to a multiplicative constant. The Sato weight of $\sigma_{\mathfrak{\natural}^{n}}(u)$ is $(n-1) g-\frac{1}{2}(n-1) n=m-(g-n)$. By Propositions 5.1 and 3.4(ii) and (i), we see that the expansion of $\sigma_{\mathfrak{\natural}^{n}}(u)$ contains a term whose coefficient is 1 or -1 . Therefore, the multiplicative constant above must be a 
polynomial of $\lambda_{0}(=1), \ldots, \lambda_{2 g+1}$. Hence the Sato weight of $\varphi^{\left(j_{0}\right)}(u)$ is at least $m-(g-n)$, so that $j_{0} \geqslant g-n$. Therefore, we have identically

$$
\varphi^{(j)}(u)=0 \quad \text { for } j=0, \ldots, g-n-1 .
$$

Because we have already found at least $n$ zeros of $v \mapsto \sigma_{\natural^{n+1}}\left(u^{(1)}+\cdots+u^{(n)}+v\right)$ for $v \neq 0$, it is impossible that $\varphi^{(g-n)}\left(u^{(1)}+\cdots+u^{(n)}\right)=0$ identically. So we see $j_{0}=g-n$ and have

$$
\sigma_{\mathfrak{\natural}^{n+1}}(u+v)=\varphi^{(g-n)}(u) v_{g}^{g-n}+\left(d^{\circ}\left(v_{g}\right) \geqslant g\right)
$$

for the non-trivial function $\varphi^{(g-n)}(u)$. Thus, we see that

$$
\varphi^{(g-n)}(u)=(-1)^{(g-n)(g-n-1) / 2} \sigma_{\natural^{n}}(u)
$$

for $u \in \kappa^{-1}\left(\Theta^{[n]}\right)$ by Proposition 3.4(ii) and Corollary 5.2. Now all the other statements are clear.

Lemma 7.6. Let $u \in \kappa^{-1}\left(\Theta^{[1]}\right)$. Then

$$
\sigma_{b}(2 u)=(-1)^{g-1} 2 u_{g}{ }^{2 g-1}+\left(d^{\circ}\left(u_{g}\right) \geqslant 2 g+1\right) .
$$

Proof. The statement follows from Proposition 3.4(iii), Corollary 5.2 and Lemma 4.3.

Lemma 7.7. Let $u \in \kappa^{-1}\left(\Theta^{[1]}\right)$. Then

$$
\frac{\sigma_{b}(2 u)}{\sigma_{\sharp}(u)^{4}}=(-1)^{g} 2 y(u) .
$$

Proof. Lemma 7.3 shows that the left-hand side is periodic with respect to $\Lambda$, and is an odd function by Lemma 4.3. We have by Lemma 7.6 that

$$
\begin{aligned}
\frac{\sigma_{b}(2 u)}{\sigma_{\sharp}(u)^{4}} & =\frac{(-1)^{g-1} 2 u_{g}{ }^{2 g-1}+\left(d^{\circ}\left(u_{g}\right) \geqslant 2 g+1\right)}{\left(u_{g}^{g}+\left(d^{\circ}\left(u_{g}\right) \geqslant g+2\right)\right)^{4}} \\
& =(-1)^{g-1} \frac{2}{u_{g}^{2 g+1}}+\cdots \\
& =(-1)^{g} 2 y(u) .
\end{aligned}
$$

\section{Frobenius-Stickelberger-type formulae}

The initial case of our Frobenius-Stickelberger-type formulae is as follows.

Lemma 8.1. Suppose that $u$ and $v$ are in $\kappa^{-1}\left(\Theta^{[1]}\right)$. We have

$$
(-1)^{g+1} \frac{\sigma_{b}(u+v) \sigma_{b}(u-v)}{\sigma_{\sharp}(u)^{2} \sigma_{\sharp}(v)^{2}}=-x(u)+x(v) \quad\left(=\left|\begin{array}{cc}
1 & x(u) \\
1 & x(v)
\end{array}\right|\right) .
$$


Proof. As a function of $u$ (or $v$ ), we see that the left-hand side is periodic with respect to $\Lambda$, by Lemma 7.3. Moreover, we see that the left-hand side has its only pole at $u=(0,0, \ldots, 0)$ modulo $\Lambda$ by Proposition $7.5(\mathrm{i})$. Proposition 7.5 also shows that the Laurent expansion of the left-hand side is of the form

$$
\frac{\left(\sigma_{\sharp}(v) u_{g}^{g-1}+\cdots\right)\left(\sigma_{\sharp}(-v) u_{g}^{g-1}+\cdots\right)}{\left(u_{g}{ }^{g}+\cdots\right)^{2} \sigma_{\sharp}(v)^{2}}=(-1)^{g} \frac{1}{u_{g}{ }^{2}}+\cdots=(-1)^{g} x(u)+\cdots .
$$

Here we have used the fact that $\sigma_{\sharp}(-v)=(-1)^{g} \sigma_{\sharp}(v)$, which follows from Lemma 4.3. Since both sides have the same zeros at $u=v$ and $u=-v$, they coincide.

The general case of our Frobenius-Stickelberger-type formula is as follows.

Theorem 8.2. Let $n$ be a fixed integer. Suppose that $u^{(1)}, \ldots, u^{(n)}$ are variables on $\kappa^{-1}\left(\Theta^{[1]}\right)$.

(i) If $2 \leqslant n \leqslant g$, then we have

$$
\begin{aligned}
(-1)^{g+n(n+1) / 2} \frac{\sigma_{\mathfrak{\natural}^{n}}\left(u^{(1)}+\cdots+u^{(n)}\right)}{\sigma_{\sharp}\left(u^{(1)}\right)^{n} \cdots \sigma_{\sharp}\left(u^{(n)}\right)^{n}} & \\
= & \left|\begin{array}{ccccc}
1 & x\left(u^{(1)}\right) & x^{2}\left(u^{(1)}\right) & \cdots & x^{n-1}\left(u^{(1)}\right) \\
1 & x\left(u^{(2)}\right) & x^{2}\left(u^{(2)}\right) & \cdots & x^{n-1}\left(u^{(2)}\right) \\
\vdots & \vdots & \vdots & \ddots & \vdots \\
1 & x\left(u^{(n)}\right) & x^{2}\left(u^{(n)}\right) & \cdots & x^{n-1}\left(u^{(n)}\right)
\end{array}\right| .
\end{aligned}
$$

(ii) If $n \geqslant g+1$, then we have

$$
\begin{aligned}
& (-1)^{(2 n-g)(g-1) / 2} \frac{\sigma\left(u^{(1)}+\cdots+u^{(n)}\right) \prod_{i<j} \sigma_{b}\left(u^{(i)}-u^{(j)}\right)}{\sigma_{\sharp}\left(u^{(1)}\right)^{n} \cdots \sigma_{\sharp}\left(u^{(n)}\right)^{n}} \\
& =\mid \begin{array}{cccc}
1 & x\left(u^{(1)}\right) & x^{2}\left(u^{(1)}\right) & \ldots \\
1 & x\left(u^{(2)}\right) & x^{2}\left(u^{(2)}\right) & \ldots \\
\vdots & \vdots & \vdots & \ddots \\
1 & x\left(u^{(n)}\right) & x^{2}\left(u^{(n)}\right) & \cdots
\end{array} \\
& x^{g}\left(u^{(1)}\right) \quad y\left(u^{(1)}\right) \quad x^{g+1}\left(u^{(1)}\right) \quad x y\left(u^{(1)}\right) \quad x^{g+2}\left(u^{(1)}\right) \quad \cdots \\
& \begin{array}{llllll}
x^{g}\left(u^{(2)}\right) & y\left(u^{(2)}\right) & x^{g+1}\left(u^{(2)}\right) & x y\left(u^{(2)}\right) & x^{g+2}\left(u^{(2)}\right) & \cdots
\end{array} \\
& \begin{array}{cccccc}
\vdots & \vdots & \vdots & \vdots & \vdots & \ddots \\
x^{g}\left(u^{(n)}\right) & y\left(u^{(n)}\right) & x^{g+1}\left(u^{(n)}\right) & x y\left(u^{(n)}\right) & x^{g+2}\left(u^{(n)}\right) & \cdots
\end{array}
\end{aligned}
$$

where the right-hand side is an $n \times n$ determinant.

Proof. While this theorem is proved using Proposition 7.5 and the same argument as in $[\mathbf{1 9}, \mathbf{2 0}]$, we give the details here because of complexity of the signature.

We prove (i) first by three steps as follows. 
Step 1. Lemma 7.3 shows both sides in (i) are periodic functions of $u^{(n)}$ with respect to $A$. Hence we regard them as functions on $C$ and will compare their divisors.

Step 2. We are still regarding both sides as functions of $u^{(n)}$. By continuity, we may assume that $u^{(1)}, \ldots, u^{(n-1)}$ are pairwise different. The right-hand side vanishes at $u^{(1)}, \ldots, u^{(n-1)}$ by Proposition 7.5(ii). The left-hand side has zeros of order 1 at the same points because of the product of $\sigma_{b}$ s. We see that the left-hand side has a pole only at $u^{(n)}=(0,0, \ldots, 0)$ modulo $\Lambda$ and its order is $(g-n+1)+(g-1)(n-1)-g n=2(n-1)$ by Proposition 7.5(i). Obviously, the right-hand side has also a pole only at $u^{(n)}=(0, \ldots, 0)$ modulo $\Lambda$. Because the lowest term of its Laurent expansion is coming only from the $(n, n)$-entry, the pole is also of order $2(n-1)$. Let $v^{(1)}, \ldots, v^{(n-1)}$ be the other zeros of the left-hand side than $u^{(1)}, \ldots, u^{(n-1)}$. As we also consider their multiplicities, it may happen that some of $v^{(1)}, \ldots, v^{(n-1)}$ coincide with some of $u^{(1)}, \ldots, u^{(n-1)}$. Anyway, the Abel-Jacobi theorem shows that

$$
u^{(1)}+\cdots+u^{(n-1)}+v^{(1)}+\cdots+v^{(n-1)} \in \Lambda .
$$

We denote this element by $\ell$. Then Lemma 7.3 states that

$$
\sigma_{\natural^{n}}\left(u^{(1)}+\cdots+u^{(n-1)}+u^{(n)}\right)=\sigma_{\natural^{n}}\left(u^{(n)}-v^{(1)}-\cdots-v^{(n-1)}\right) \chi(\ell) \exp L\left(u+\frac{1}{2} \ell, \ell\right) .
$$

Hence this function of $u^{(n)}$ has zeros at $v^{(1)}, \ldots, v^{(n-1)}$ of order 1 by Proposition 7.5(ii). Therefore, the two sides have the same divisor, and they are equal up to multiplication by a function of $u^{(1)}, \ldots, u^{(n-1)}$.

Step 3. Finally, we check that the undetermined function above is 1 by comparing the coefficients of the lowest term in the Laurent expansion with respect to $u^{(n)}$. The expansion of the left-hand side is

$$
\begin{aligned}
& {\left[\left\{(-1)^{(g-n+1)(g-n) / 2} \sigma_{\sharp^{n-1}}\left(u^{(1)}+\cdots+u^{(n-1)}\right) v_{g}{ }^{g-n+1}+\cdots\right\}\right.} \\
& \left.\times \prod_{i<j \leqslant n-1} \sigma_{b}\left(u^{(i)}-u^{(j)}\right) \prod_{i=1}^{n-1}\left\{(-1)^{(g-1)(g-2) / 2} \sigma_{\sharp}\left(u^{(i)}\right)\left(-u_{g}^{(n)}\right)^{g-1}+\cdots\right\}\right] \\
& {\left[\sigma_{\sharp}\left(u^{(1)}\right)^{n} \cdots \sigma_{\sharp}\left(u^{(n-1)}\right)^{n}\left\{(-1)^{(g-2)(g-3) / 2}\left(u_{g}^{(n)}\right)^{g}+\cdots\right\}^{n}\right]+\cdots} \\
& =(-1)^{(g-n+1)(g-n) / 2+(g-1)(g-2)(n-1) / 2+(g-1)-(g-2)(g-3) n / 2} \\
& \quad \times \frac{\sigma_{\mathfrak{\natural}^{n-1}}\left(u^{(1)}+\cdots+u^{(n-1)}\right) \prod_{i<j \leqslant n-1} \sigma_{b}\left(u^{(i)}-u^{(j)}\right)}{\sigma_{\sharp}\left(u^{(1)}\right)^{n-1} \cdots \sigma_{\sharp}\left(u^{(n-1)}\right)^{n-1}} \frac{1}{\left(u_{g}^{(n)}\right)^{2(n-1)}}+\cdots .
\end{aligned}
$$

Here we see easily that the index of $(-1)$ is given by

$$
\begin{aligned}
\frac{1}{2}(g-n+1)(g-n)+\frac{1}{2}(g-1)(g-2)(n-1)+(g-1)-\frac{1}{2}(g-2)(g-3) n & \\
& \equiv g+\frac{1}{2}(n-1) n \bmod 2 .
\end{aligned}
$$

The expansion

$$
\left|\begin{array}{ccccc}
1 & x\left(u^{(1)}\right) & x^{2}\left(u^{(1)}\right) & \cdots & x^{n-2}\left(u^{(1)}\right) \\
1 & x\left(u^{(2)}\right) & x^{2}\left(u^{(2)}\right) & \cdots & x^{n-2}\left(u^{(2)}\right) \\
\vdots & \vdots & \vdots & \ddots & \vdots \\
1 & x\left(u^{(n-1)}\right) & x^{2}\left(u^{(n-1)}\right) & \cdots & x^{n-2}\left(u^{(n-1)}\right)
\end{array}\right| x^{n-1}\left(u^{(n)}\right)+\cdots
$$


of the right-hand side with $x\left(u^{(n)}\right)=1 /\left(u^{(n)}\right)^{2}+\cdots$ and the induction hypothesis shows that the coefficients coincide. Thus, we have proved (i).

Now let us prove (ii). While the argument is entirely similar to that of (i), we describe it explicitly again, in order to enable the reader to check the signature of the desired formulae more easily. Regarding both sides to be functions of $u^{(n)}$ again, the formulae corresponding to the Steps 1 and 2 above are proved similarly. So, we omit them. To prove the part corresponding to Step 3 above, we note that the leading term of the Laurent expansion of the right-hand side contains the signature

$$
\begin{aligned}
& \frac{(-1)^{(n+g-1)}}{\left(u_{g}^{(n)}\right)^{n+g-1}} \\
& \quad \times\left|\begin{array}{cccccccc}
1 & x\left(u^{(1)}\right) & \cdots & x^{g}\left(u^{(1)}\right) & y\left(u^{(1)}\right) & x^{g+1}\left(u^{(1)}\right) & x y\left(u^{(1)}\right) & \cdots \\
1 & x\left(u^{(2)}\right) & \cdots & x^{g}\left(u^{(2)}\right) & y\left(u^{(2)}\right) & x^{g+1}\left(u^{(2)}\right) & x y\left(u^{(2)}\right) & \cdots \\
\vdots & \vdots & \ddots & \vdots & \vdots & \vdots & \vdots & \ddots \\
1 & x\left(u^{(n-1)}\right) & \cdots & x^{g}\left(u^{(n-1)}\right) & y\left(u^{(n-1)}\right) & x^{g+1}\left(u^{(n-1)}\right) & x y\left(u^{(n-1)}\right) & \cdots
\end{array}\right| .
\end{aligned}
$$

Expanding the left-hand side gives

$$
\begin{aligned}
& {\left[\left\{\sigma\left(u^{(1)}+\cdots+u^{(n-1)}\right)+\cdots\right\}\right.} \\
& \left.\times \prod_{i<j \leqslant n-1} \sigma_{b}\left(u^{(i)}-u^{(j)}\right) \prod_{i=1}^{n-1}\left\{(-1)^{(g-1)(g-2) / 2} \sigma_{\sharp}\left(u^{(i)}\right)\left(-u_{g}^{(n)}\right)^{g-1}+\cdots\right\}\right] \\
& {\left[\sigma_{\sharp}\left(u^{(1)}\right)^{n} \cdots \sigma_{\sharp}\left(u^{(n-1)}\right)^{n}\left\{\left((-1)^{(g-2)(g-3) / 2}\left(u_{g}^{(n)}\right)^{g}\right)^{n}+\cdots\right\}\right]} \\
& =(-1)^{(g-1)(g-2)(n-1) / 2+(g-1)-(g-2)(g-3) n / 2} \\
& \quad \times \frac{\sigma_{\mathfrak{\natural}^{n-1}}\left(u^{(1)}+\cdots+u^{(n-1)}\right) \prod_{i<j \leqslant n-1} \sigma_{b}\left(u^{(i)}-u^{(j)}\right)}{\sigma_{\sharp}\left(u^{(1)}\right)^{n-1} \cdots \sigma_{\sharp}\left(u^{(n-1)}\right)^{n-1}} \frac{1}{\left(u_{g}^{(n)}\right)^{n+g-1}}+\cdots .
\end{aligned}
$$

Because the index of the quotient of the signatures of the two leading terms above is given by

$$
\begin{aligned}
\frac{1}{2}(g-1)(g-2)(n-1)+(g-1)-\frac{1}{2}(g-2)(g-3) n-(n+g-1) & \\
& \equiv \frac{1}{2}(2 n-2-g)(g-1) \bmod 2,
\end{aligned}
$$

we can use the induction hypothesis to obtain (ii).

\section{Kiepert-type formulae}

The function $\sigma(u)$ is directly related to $x(u)$ as follows.

Lemma 9.1. Fix $j$ with $0 \leqslant j \leqslant g$. Let $u$ and $v$ be on $\kappa^{-1}\left(\Theta^{[1]}\right)$. Then

$$
\lim _{u \rightarrow v} \frac{\sigma_{b}(u-v)}{u_{j}-v_{j}}=\frac{1}{x^{j-1}(v)} .
$$


Proof. Because of Lemma 8.1 we have

$$
\frac{x(u)-x(v)}{u_{j}-v_{j}}=(-1)^{g} \frac{\sigma_{b}(u+v)}{\sigma_{\sharp}(u)^{2} \sigma_{\sharp}(v)^{2}} \frac{\sigma_{b}(u-v)}{u_{j}-v_{j}} .
$$

Now we let $u$ tend to $v$. Then the limit of the left-hand side is

$$
\lim _{u \rightarrow v} \frac{x(u)-x(v)}{u_{j}-v_{j}}=\frac{\mathrm{d} x}{\mathrm{~d} u_{j}}(v) .
$$

This is equal to $2 y / x^{j-1}(v)$ by the definition. The required formula follows from Lemma 7.7.

Definition 9.2. For $u \in \kappa^{-1}\left(\Theta^{[1]}\right)$ we denote by $\psi_{n}(u)$ the function $\sigma_{\natural^{n}}(n u) / \sigma_{\sharp}(u)^{n^{2}}$ if $n \leqslant g$, and $\sigma(n u) / \sigma_{\sharp}(u)^{n^{2}}$ if $n \geqslant g+1$.

This function $\psi_{n}(u)$ has the following expression, which is a natural generalization of the classical formula of Kiepert [14].

Theorem 9.3 (Kiepert-type formula). Let $u \in \kappa^{-1}\left(\Theta^{[1]}\right)$ and let $n$ be a positive integer.

(i) If $1 \leqslant n \leqslant g$, then $\psi_{n}(u)=(-1)^{g+n(n+1) / 2}(2 y(u))^{n(n-1) / 2}$.

(ii) We fix $j$ with $1 \leqslant j \leqslant g$. If $n \geqslant g+1$, then we have

$$
\begin{aligned}
& (-1)^{(2 n-g)(g-1) / 2}(1 ! 2 ! \cdots(n-1) !) \psi_{n}(u)=x^{(j-1) n(n-1) / 2}(u) \\
& \times \mid \begin{array}{ccc}
x^{\prime} & \left(x^{2}\right)^{\prime} & \cdots \\
x^{\prime \prime} & \left(x^{2}\right)^{\prime \prime} & \cdots \\
x^{\prime \prime \prime} & \left(x^{2}\right)^{\prime \prime \prime} & \cdots \\
\vdots & \vdots & \ddots \\
x^{(n-1)} & \left(x^{2}\right)^{(n-1)} & \cdots
\end{array} \\
& \left.\begin{array}{cccccc}
\left(x^{g}\right)^{\prime} & y^{\prime} & \left(x^{g+1}\right)^{\prime} & (y x)^{\prime} & \left(x^{g+2}\right)^{\prime} & \ldots \\
\left(x^{g}\right)^{\prime \prime} & y^{\prime \prime} & \left(x^{g+1}\right)^{\prime \prime} & (y x)^{\prime \prime} & \left(x^{g+2}\right)^{\prime \prime} & \ldots \\
\left(x^{3}\right)^{\prime \prime \prime} & y^{\prime \prime \prime} & \left(x^{g+1}\right)^{\prime \prime \prime} & (y x)^{\prime \prime \prime} & \left(x^{g+2}\right)^{\prime \prime \prime} & \ldots \\
\vdots & \vdots & \vdots & \vdots & \vdots & \ddots \\
\left(x^{g}\right)^{(n-1)} & y^{(n-1)} & \left(x^{g+1}\right)^{(n-1)} & (y x)^{(n-1)} & \left(x^{g+2}\right)^{(n-1)} & \ldots
\end{array}\right) .
\end{aligned}
$$

where the size of the matrix is $(n-1) \times(n-1)$, and the symbols ${ }^{\prime},{ }^{\prime \prime}, \ldots,{ }^{(n-1)}$ denote

$$
\frac{\mathrm{d}}{\mathrm{d} u_{j}},\left(\frac{\mathrm{d}}{\mathrm{d} u_{j}}\right)^{2}, \ldots,\left(\frac{\mathrm{d}}{\mathrm{d} u_{j}}\right)^{n-1} .
$$

Proof. If $1 \leqslant n \leqslant g+1$, the right-hand side of Theorem 8.2 is a Vandermonde determinant. Hence we have statement (i), by using Lemma 9.1. Statement (ii) is proved by the same argument as in [19], using Theorem 8.2 and Lemma 9.1. 
Remark 9.4. The polynomials $\psi_{n}(u)$ are the natural generalization of division polynomials of an elliptic curve, and are used to find torsion points on the curve $C$ in the Jacobian variety $J$. Indeed, for $n \geqslant g, u \in \kappa^{-1} \iota(C)$ is an $n$-torsion in $J$ if and only if all of $\psi_{n-g+1}(u), \psi_{n-g+2}(u), \ldots, \psi_{n}(u), \ldots, \psi_{n+g-1}(u)$ vanish. A detailed description of this fact is seen in $[\mathbf{8}]$.

Finally, we mention the degree of the polynomials above.

Proposition 9.5. The number of roots, counting multiplicities, of the equation $\psi_{n}(u)=0$ is $\frac{1}{2} n(n-1)(2 g+1)$ if $1 \leqslant n \leqslant g-1$ and $n^{2} g-\frac{1}{2} g(g+1)$ if $n \geqslant g$.

Proof. The number of the roots is equal to the order of the pole at $u=(0, \ldots, 0)$. If $u \in \kappa^{-1}\left(\Theta^{[1]}\right)$, then $u_{g}$ is a local parameter at $u=(0, \ldots, 0)$ because of Lemma 4.7. We calculate it for the case $j=g$. We denote $u_{g}$ by $t$. If $n \geqslant g$, the lowest term of the Laurent expansion of the determinant in Theorem 9.3 at $u=(0,0, \ldots, 0)$ is exactly the same as that of

$$
\begin{array}{|cccc}
-\frac{2}{t^{3}} & -\frac{4}{t^{5}} & \cdots & \\
\frac{4 \cdot 3}{t^{4}} & \frac{6 \cdot 5}{t^{6}} & \cdots & \\
\vdots & \vdots & \ddots & \\
(-1)^{n-1} \frac{(n+1) \cdots 3}{t^{n+1}} & (-1)^{n-1} \frac{(n+3) \cdots 5}{t^{n+3}} & \cdots & \\
-\frac{2 g}{t^{2 g+1}} & \cdots & -\frac{n+g-1}{t^{n+g}} \\
\frac{(2 g+2)(2 g+1)}{t^{2 g+2}} & \cdots & \frac{(n+g+1)(n+g)}{t^{n+g+1}} \\
\vdots & \ddots & \vdots \\
(-1)^{n-1} \frac{(n+2 g-1) \cdots(2 g-1)}{t^{2 g+n-1}} & \cdots & (-1)^{n-1} \frac{(2 n+g-1) \cdots(n+g-1)}{t^{2 n+g-2}}
\end{array} \mid .
$$

This is equal to

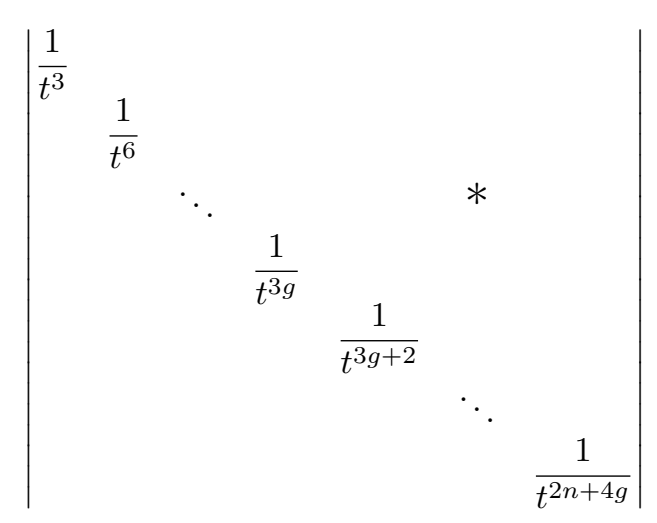


times a non-zero constant. This determinant is the $\left(\frac{3}{2} g(g+1)+(n-g-1)(n+2 g)\right)$ th power of $1 / t$. Hence we see that

$$
x^{(g-1) n(n-1) / 2}(u)=1 / t^{(g-1) n(n-1)}+\cdots .
$$

So the lowest term of the Laurent expansion of the right-hand side of the formula in Theorem 9.3 is the $\left(n^{2} g-\frac{1}{2} g(g+1)\right)$ th power of $1 / t$. If $n<g$, then the lowest term is easily seen to be the $\frac{1}{2} n(n-1)(2 g+1)$ th power of $1 / t$.

Finally, we have a remark. The derivation $D_{\infty}$ used in Mumford's book [17, p. 3.155] is essentially no other than our $\partial / \partial u_{g}$. While we could rewrite our expressions using mainly this $D_{\infty}$, the author hopes to describe such things on another occasion.

Acknowledgements. The author thanks the referee who read carefully the first version of this paper. If this final version is easier to read, this is due to the referee's suggestions. The author also thanks Shigeki Matsutani for permission to reproduce his proof of Cantor's formula below. It forms an essential part of the results presented here.

\section{Appendix A. Connection between the formulae of Cantor-Brioschi and those of Kiepert type}

In this appendix* we prove a formula of Cantor in [8] (Theorem A 1 below) by using Theorem 9.3. This is a detailed exposition of the appendix in [16]. Since our argument is reversible, Theorem 9.3 is proved using the formula of Cantor.

Let $u=\left(u_{1}, u_{2}, \ldots, u_{g}\right)$ be the system of variables explained in $\S 4$. We assume that $u$ belongs to $\kappa^{-1} \iota(C)$. So we may use the notation $x(u)$ and $y(u)$. If $\mu(u)$ is a function on $\kappa^{-1} \iota(C)$, we can regard it locally as a function of $u_{1}$. We denote by $\mu^{\prime}(u), \mu^{\prime \prime}(u), \ldots, \mu^{(\nu)}(u), \ldots$ the functions obtained by applying

$$
\frac{\mathrm{d}}{\mathrm{d} u_{1}},\left(\frac{\mathrm{d}}{\mathrm{d} u_{1}}\right)^{2}, \ldots,\left(\frac{\mathrm{d}}{\mathrm{d} u_{1}}\right)^{\nu}, \ldots
$$

to the function $\mu(u)$ along $\iota(C)$; and by $\dot{\mu}(u), \ddot{\mu}(u), \ldots, \mu^{\langle\nu\rangle}(u), \ldots$ the functions given by applying

$$
\frac{\mathrm{d}}{\mathrm{d} x},\left(\frac{\mathrm{d}}{\mathrm{d} x}\right)^{2}, \ldots,\left(\frac{\mathrm{d}}{\mathrm{d} x}\right)^{\nu}, \ldots
$$

to $\mu(u)$. Here we regard $\mu(u)$ locally as a function of $x=x(u)$.

Recall that $\psi_{n}(u)$ was defined in Definition 9.2. A determinant expression for $\psi_{n}(u)$, due to Cantor, is the following.

* By Shigeki Matsutani, 8-21-1, Higashi-Linkan Sagamihara 228-0811, Japan (rxb01142@nifty.com). 
Theorem A 1 (Cantor [8]). Suppose $n \geqslant g+2$. Let $s$ be the largest integer not exceeding $(n-g) / 2$, and $r=n-1-s$. Then

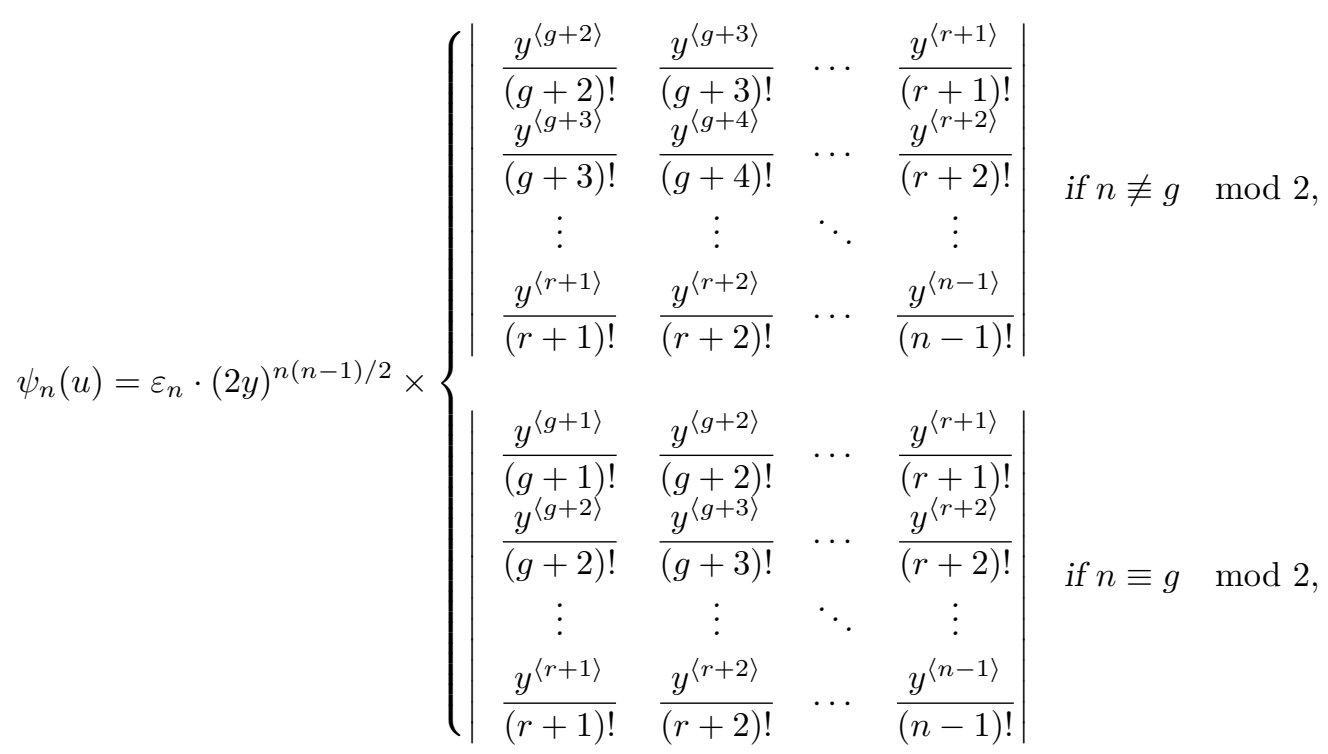

where $\varepsilon_{n}$ is determined by $g$ modulo 4 and $n$ modulo 4 as in the following table:

\begin{tabular}{crrrr}
\hline$g \bmod 4$ & $\varepsilon_{0}$ & $\varepsilon_{1}$ & $\varepsilon_{2}$ & $\varepsilon_{3}$ \\
\hline 0 & -1 & -1 & 1 & -1 \\
1 & -1 & 1 & 1 & 1 \\
2 & 1 & -1 & -1 & -1 \\
3 & 1 & 1 & -1 & 1 \\
\hline
\end{tabular}

\section{Remark A 2.}

(i) Both matrices above are $s \times s$.

(ii) The number $r$ and $s$ are just the number of entries of the form $\left(x^{k}\right)^{\prime}$ with $k \geqslant 1$ and of the form $\left(y x^{j}\right)^{\prime}$ with $j \geqslant 0$, respectively, in the first row of the determinant in Theorem 9.3(ii).

(iii) The constant factor is not clear from our definition of $\psi_{n}(u)$ in Definition 9.2 and $[\boldsymbol{8}]$. It will be determined when our calculation has been completed.

(iv) This formula for the case $g=1$ is known classically (Brioschi [4]).

(v) Since our proof depends on Theorem 8.2, it works only over the field of complex numbers.

The following lemma is easily checked. 
Lemma A 3. Let $m>0$ be any integer. One has

$$
\left(\frac{\mathrm{d}}{\mathrm{d} u_{1}}\right)^{m}=(2 y)^{m}(u)\left(\frac{\mathrm{d}}{\mathrm{d} x}\right)^{m}+\sum_{j=1}^{m} a_{j}^{(m)}(u)\left(\frac{\mathrm{d}}{\mathrm{d} x}\right)^{j} .
$$

Here $a_{j}^{(m)}(u)$ are polynomials in

$$
y(u), \frac{\mathrm{d} y}{\mathrm{~d} x}(u), \frac{\mathrm{d}^{2} y}{\mathrm{~d} x^{2}}(u), \ldots, \frac{\mathrm{d}^{m-1} y}{\mathrm{~d} x^{m-1}}(u) .
$$

Let $s=n-1-r$. Then, by Theorem 9.3 for $j=1$, we have

$$
\begin{aligned}
& c_{n}^{\prime} 1 ! 2 ! \cdots(n-1) ! \psi_{n}(u) \\
& =(-1)^{r(r+1) / 2} \\
& \quad \times\left|\begin{array}{cccccccc}
x^{\prime} & \left(x^{2}\right)^{\prime} & \ldots & \left(x^{r}\right)^{\prime} & y^{\prime} & (y x)^{\prime} & \ldots & \left(y x^{s-1}\right)^{\prime} \\
x^{\prime \prime} & \left(x^{2}\right)^{\prime \prime} & \ldots & \left(x^{r}\right)^{\prime \prime} & y^{\prime \prime} & (y x)^{\prime \prime} & \ldots & \left(y x^{s-1}\right)^{\prime \prime} \\
x^{\prime \prime \prime} & \left(x^{2}\right)^{\prime \prime \prime} & \ldots & \left(x^{r}\right)^{\prime \prime \prime} & y^{\prime \prime \prime} & (y x)^{\prime \prime \prime} & \ldots & \left(y x^{s-1}\right)^{\prime \prime \prime} \\
\vdots & \vdots & & \vdots & \vdots & \vdots & \ddots & \vdots \\
x^{(n-1)} & \left(x^{2}\right)^{(n-1)} & \ldots & \left(x^{r}\right)^{(n-1)} & y^{(n-1)} & (y x)^{(n-1)} & \ldots & \left(y x^{s-1}\right)^{(n-1)}
\end{array}\right|(u) .
\end{aligned}
$$

Here the determinant is $(n-1) \times(n-1)$. By Lemma A 3 we have that

$$
\left[\begin{array}{c}
\frac{\mathrm{d}}{\mathrm{d} u_{1}} \\
\left(\frac{\mathrm{d}}{\mathrm{d} u_{1}}\right)^{2} \\
\left(\frac{\mathrm{d}}{\mathrm{d} u_{1}}\right)^{3} \\
\vdots \\
\left(\frac{\mathrm{d}}{\mathrm{d} u_{1}}\right)^{n-1}
\end{array}\right]=\left[\begin{array}{ccccc}
2 y & & & & \\
a_{2}^{(1)} & (2 y)^{2} & & & \\
a_{3}^{(1)} & a_{3}^{(2)} & (2 y)^{3} & & \\
\vdots & \vdots & \vdots & \ddots & \\
a_{n-1}^{(1)} & a_{n-1}^{(2)} & a_{n-1}^{(3)} & \cdots & (2 y)^{n-1}
\end{array}\right]\left[\begin{array}{c}
\frac{\mathrm{d}}{\mathrm{d} x} \\
\left(\frac{\mathrm{d}}{\mathrm{d} x}\right)^{2} \\
\left(\frac{\mathrm{d}}{\mathrm{d} x}\right)^{3} \\
\vdots \\
\left(\frac{\mathrm{d}}{\mathrm{d} x}\right)^{n-1}
\end{array}\right]
$$

Now we consider

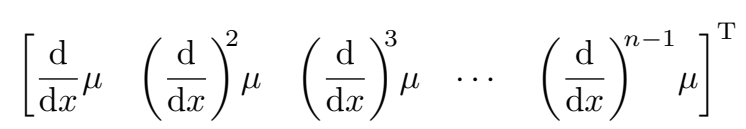


for $\mu=x, x^{2}, \ldots$ and $y, y x, y x^{2}, \ldots$ Obviously,

$$
\left[\begin{array}{c}
\frac{\mathrm{d}}{\mathrm{d} x} \\
\left(\frac{\mathrm{d}}{\mathrm{d} x}\right)^{2} \\
\left(\frac{\mathrm{d}}{\mathrm{d} x}\right)^{3} \\
\vdots \\
\left(\frac{\mathrm{d}}{\mathrm{d} x}\right)^{n-1}
\end{array}\right]\left[\begin{array}{llll}
x & x^{2} & \cdots & x^{r}
\end{array}\right]=\left[\begin{array}{lllll}
1 ! & & & * & \\
& 2 ! & & & \\
& 3 ! & & \\
& & \ddots & \\
& & & & \\
& & & & \\
& & & &
\end{array}\right]
$$

For $\mu=y, y x, \ldots, y x^{s-1}$, we have that

$$
\left[\begin{array}{c}
\frac{\mathrm{d}}{\mathrm{d} x} \\
\left(\frac{\mathrm{d}}{\mathrm{d} x}\right)^{2} \\
\left(\frac{\mathrm{d}}{\mathrm{d} x}\right)^{3} \\
\vdots \\
\left(\frac{\mathrm{d}}{\mathrm{d} x}\right)^{n-1}
\end{array}\right]\left[\begin{array}{lllll}
y & y x & y x^{2} & \cdots & y x^{s-1}
\end{array}\right]
$$

is equal to

$$
\left.\begin{array}{ccc}
{\left[\begin{array}{c}
1 \\
0
\end{array}\right) \dot{y}} & \left(\begin{array}{l}
1 \\
0
\end{array}\right) \dot{y} x+\left(\begin{array}{l}
1 \\
1
\end{array}\right) y & \left(\begin{array}{l}
1 \\
0
\end{array}\right) \dot{y} x^{2}+\left(\begin{array}{l}
1 \\
1
\end{array}\right) y \cdot 2 x \\
\left(\begin{array}{l}
2 \\
0
\end{array}\right) \ddot{y} & \left(\begin{array}{l}
2 \\
0
\end{array}\right) \ddot{y} x+\left(\begin{array}{l}
2 \\
1
\end{array}\right) \dot{y} & \left(\begin{array}{l}
2 \\
0
\end{array}\right) \ddot{y} x^{2}+\left(\begin{array}{l}
2 \\
1
\end{array}\right) \dot{y} \cdot 2 x+\left(\begin{array}{l}
2 \\
2
\end{array}\right) y \cdot 2 ! \\
\left(\begin{array}{l}
3 \\
0
\end{array}\right) \dddot{y} & \left(\begin{array}{l}
3 \\
0
\end{array}\right) \dddot{y} x+\left(\begin{array}{l}
3 \\
1
\end{array}\right) \ddot{y} & \left(\begin{array}{l}
3 \\
0
\end{array}\right) \dddot{y} x^{2}+\left(\begin{array}{l}
3 \\
1
\end{array}\right) \ddot{y} \cdot 2 x+\left(\begin{array}{l}
3 \\
2
\end{array}\right) \dot{y} \cdot 2 ! \\
\vdots & \vdots & \ldots \\
& \left(\begin{array}{l}
1 \\
0
\end{array}\right) \dot{y} x^{3}+\left(\begin{array}{l}
1 \\
1
\end{array}\right) y \cdot 3 x^{2} & \\
\left(\begin{array}{l}
2 \\
0
\end{array}\right) \ddot{y} x^{3}+\left(\begin{array}{l}
2 \\
1
\end{array}\right) \dot{y} \cdot 3 x^{2}+\left(\begin{array}{l}
2 \\
2
\end{array}\right) y \cdot 3 \cdot 2 x & \ldots \\
\left(\begin{array}{l}
3 \\
0
\end{array}\right) \dddot{y} x^{3}+\left(\begin{array}{l}
3 \\
1
\end{array}\right) \ddot{y} \cdot 3 x^{2}+\left(\begin{array}{l}
3 \\
2
\end{array}\right) \dot{y} \cdot 3 \cdot 2 x+\left(\begin{array}{l}
3 \\
3
\end{array}\right) y \cdot 3 ! & \ldots \\
\vdots & \ddots
\end{array}\right],
$$


which is equal to

$$
\begin{aligned}
& \left(\begin{array}{ccccc}
\left(\begin{array}{c}
1 \\
0
\end{array}\right) \frac{\mathrm{d}}{\mathrm{d} x} & \left(\begin{array}{l}
1 \\
1
\end{array}\right) & & & \\
\left(\begin{array}{l}
2 \\
0
\end{array}\right)\left(\frac{\mathrm{d}}{\mathrm{d} x}\right)^{2} & \left(\begin{array}{l}
2 \\
1
\end{array}\right) \frac{\mathrm{d}}{\mathrm{d} x} & \left(\begin{array}{l}
2 \\
2
\end{array}\right) & & \\
\vdots & \vdots & \vdots & \ddots & \\
\left(\begin{array}{c}
s-1 \\
0
\end{array}\right)\left(\frac{\mathrm{d}}{\mathrm{d} x}\right)^{s-1} & \left(\begin{array}{c}
s-1 \\
1
\end{array}\right)\left(\frac{\mathrm{d}}{\mathrm{d} x}\right)^{s-2} & \left(\begin{array}{c}
s-1 \\
2
\end{array}\right)\left(\frac{\mathrm{d}}{\mathrm{d} x}\right)^{s-3} & \cdots & \left(\begin{array}{l}
s-1 \\
s-1
\end{array}\right) \\
\vdots & \vdots & \vdots & \ddots & \vdots \\
\left(\begin{array}{c}
r \\
0
\end{array}\right)\left(\frac{\mathrm{d}}{\mathrm{d} x}\right)^{r} & \left(\begin{array}{c}
r \\
1
\end{array}\right)\left(\frac{\mathrm{d}}{\mathrm{d} x}\right)^{r-1} & \left(\begin{array}{c}
r \\
2
\end{array}\right)\left(\frac{\mathrm{d}}{\mathrm{d} x}\right)^{r-2} & \cdots & \left(\begin{array}{c}
r \\
s-1
\end{array}\right)\left(\frac{\mathrm{d}}{\mathrm{d} x}\right)^{r-s+1} \\
\hline\left(\begin{array}{c}
r+1 \\
0
\end{array}\right)\left(\frac{\mathrm{d}}{\mathrm{d} x}\right)^{r+1} & \left(\begin{array}{c}
r+1 \\
1
\end{array}\right)\left(\frac{\mathrm{d}}{\mathrm{d} x}\right)^{r} & \left(\begin{array}{c}
r+1 \\
2
\end{array}\right)\left(\frac{\mathrm{d}}{\mathrm{d} x}\right)^{r-1} & \cdots & \left(\begin{array}{c}
r+1 \\
s-1
\end{array}\right)\left(\frac{\mathrm{d}}{\mathrm{d} x}\right)^{r-s+2} \\
\vdots & \vdots & \vdots & \ddots & \vdots \\
\left(\begin{array}{c}
n-1 \\
0
\end{array}\right)\left(\frac{\mathrm{d}}{\mathrm{d} x}\right)^{n-1} & \left(\begin{array}{c}
n-1 \\
1
\end{array}\right)\left(\frac{\mathrm{d}}{\mathrm{d} x}\right)^{n-2} & \left(\begin{array}{c}
n-1 \\
2
\end{array}\right)\left(\frac{\mathrm{d}}{\mathrm{d} x}\right)^{n-3} & \cdots & \left(\begin{array}{c}
n-1 \\
s-1
\end{array}\right)\left(\frac{\mathrm{d}}{\mathrm{d} x}\right)^{n-s}
\end{array}\right] \\
& \times\left.\left[\begin{array}{ccccc}
y & y T & y \cdot T^{2} & \cdots & y \cdot T^{s-1} \\
& y & y \cdot 2 T & \cdots & y \cdot(s-1) T^{s-2} \\
& & y \cdot 2 ! & \cdots & y \cdot(s-1)(s-2) T^{s-3} \\
& & & \ddots & \vdots \\
& & & & y \cdot(s-1) !
\end{array}\right]\right|_{T=x}
\end{aligned}
$$

Thus

$$
\operatorname{det}\left(\left[\begin{array}{c}
\frac{\mathrm{d}}{\mathrm{d} x} \\
\left(\frac{\mathrm{d}}{\mathrm{d} x}\right)^{2} \\
\left(\frac{\mathrm{d}}{\mathrm{d} x}\right)^{3} \\
\vdots \\
\left(\frac{\mathrm{d}}{\mathrm{d} x}\right)^{n-1}
\end{array}\right]\left[\begin{array}{llllllll}
x & x^{2} & \cdots & x^{r} & y & y x & \cdots & y x^{s-1}
\end{array}\right]\right)
$$

is equal to $(1 ! 2 ! \cdots r !)$ times

$$
\operatorname{det}\left(\left[\begin{array}{ccc}
\left(\begin{array}{c}
r+1 \\
0
\end{array}\right)\left(\frac{\mathrm{d}}{\mathrm{d} x}\right)^{r+1} & \cdots & \left(\begin{array}{c}
r+1 \\
s-1
\end{array}\right)\left(\frac{\mathrm{d}}{\mathrm{d} x}\right)^{r-s+2} \\
\vdots & \ddots & \vdots \\
\left(\begin{array}{c}
n-1 \\
0
\end{array}\right)\left(\frac{\mathrm{d}}{\mathrm{d} x}\right)^{n-1} & \cdots & \left(\begin{array}{c}
n-1 \\
s-1
\end{array}\right)\left(\frac{\mathrm{d}}{\mathrm{d} x}\right)^{n-s}
\end{array}\right]\right.
$$




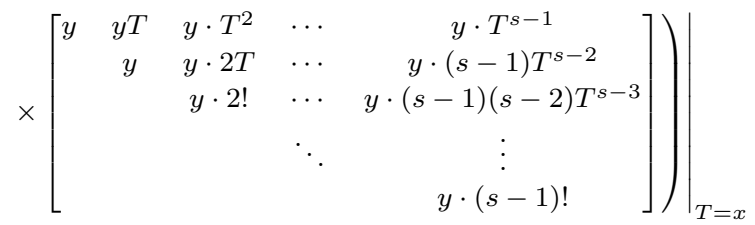

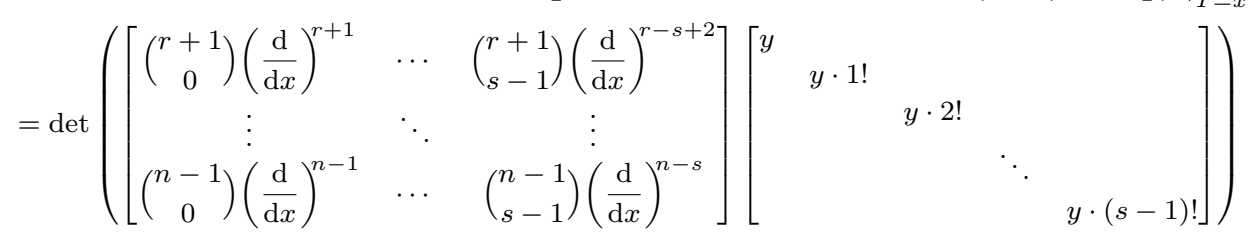

$$
\begin{aligned}
& =\operatorname{det}\left(\left[\begin{array}{cccc}
\left(\begin{array}{c}
r+1 \\
0
\end{array}\right)\left(\frac{\mathrm{d}}{\mathrm{d} x}\right)^{r+1} & 1 !\left(\begin{array}{c}
r+1 \\
1
\end{array}\right)\left(\frac{\mathrm{d}}{\mathrm{d} x}\right)^{r} & \cdots & (s-1) !\left(\begin{array}{c}
r+1 \\
s-1
\end{array}\right)\left(\frac{\mathrm{d}}{\mathrm{d} x}\right)^{r-s+2} \\
\left(\begin{array}{c}
r+2 \\
0
\end{array}\right)\left(\frac{\mathrm{d}}{\mathrm{d} x}\right)^{r+2} & 1 !\left(\begin{array}{c}
r+2 \\
1
\end{array}\right)\left(\frac{\mathrm{d}}{\mathrm{d} x}\right)^{r+1} & \cdots & (s-1) !\left(\begin{array}{c}
r+2 \\
s-1
\end{array}\right)\left(\frac{\mathrm{d}}{\mathrm{d} x}\right)^{r-s+3} \\
\vdots & \vdots & \ddots & \vdots \\
\left(\begin{array}{c}
n-1 \\
0
\end{array}\right)\left(\frac{\mathrm{d}}{\mathrm{d} x}\right)^{n-1} & 1 !\left(\begin{array}{c}
n-1 \\
1
\end{array}\right)\left(\frac{\mathrm{d}}{\mathrm{d} x}\right)^{n-2} & \cdots & (s-1) !\left(\begin{array}{c}
n-1 \\
s-1
\end{array}\right)\left(\frac{\mathrm{d}}{\mathrm{d} x}\right)^{n-s}
\end{array}\right]\right.
\end{aligned}
$$

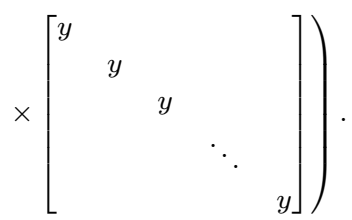

By dividing the first row by $(r+1)$ !, the second row by $(r+2)$ !, and so on, we see that this determinant is equal to

$$
(r+1) !(r+2) ! \cdots(n-1) !\left|\begin{array}{cccc}
\frac{y^{\langle r+1\rangle}}{(r+1) !} & \frac{y^{\langle r\rangle}}{r !} & \cdots & \frac{y^{\langle r-s+2\rangle}}{(r-s+2) !} \\
\frac{y^{\langle r+2\rangle}}{(r+2) !} & \frac{y^{\langle r+1\rangle}}{(r+1) !} & \cdots & \frac{y^{\langle r-s+3\rangle}}{(r-s+3) !} \\
\vdots & \vdots & \ddots & \vdots \\
\frac{y^{\langle n-1\rangle}}{(n-1) !} & \frac{y^{\langle n-2\rangle}}{(n-2) !} & \cdots & \frac{y^{\langle n-s\rangle}}{(n-s) !}
\end{array}\right| .
$$

If we reorder the columns (or the rows) of this determinant, we have the right-hand side of Theorem A 1 with the signature $(-1)^{s(s-1) / 2}$. Then the total signature is given by

$$
\begin{aligned}
(-1)^{(2 n-g)(g-1) / 2} \cdot(-1)^{s(s-1) / 2+r(r-1) / 2} & \\
& =(-1)^{(2 n-g)(g-1) / 2} \cdot(-1)^{-s(s-1) / 2+r(r-1) / 2} \\
& =(-1)^{(2 n-g)(g-1) / 2} \cdot(-1)^{(r-s)(r+s) / 2-(r-s) / 2} \\
& =(-1)^{(2 n-g)(g-1) / 2} \cdot(-1)^{(r-s)(n-2) / 2} \\
& =(-1)^{(2 n-g)(g-1) / 2} \cdot \begin{cases}(-1)^{g(n-2) / 2} & \text { if } n \not \equiv g \bmod 2, \\
(-1)^{(g-1)(n-2) / 2} & \text { if } n \equiv g \bmod 2 .\end{cases}
\end{aligned}
$$


Computing this for $n$ and $g$ modulo 4 yields the value of $\varepsilon_{n}$ in the table in Theorem A 1 , and the desired formulae have been obtained.

\section{References}

1. E. Arbarello, M. Cornalba, P. A. Griffiths and J. Harris, Geometry of algebraic curves, vol. 1, Grundlehren der mathematischen Wissenschaften, vo. 267 (Springer, 1984).

2. H. F. BAKER, On the hyperelliptic sigma functions, Am. J. Math. 20 (1898), 301-384.

3. H. F. BAKER, Abelian functions-Abel's theorem and the allied theory including the theory of the theta functions (Cambridge University Press, 1897; reprint, 1995).

4. F. BRIOSCHI, Sur quelques formules pour la multiplication des fonctions elliptiques, $C$. R. Acad. Sci. Paris 59 (1864), 769-775.

5. V. M. Buchstaber, V. Z. Enolski and D. V. Leykin, Kleinian functions, hyperelliptic Jacobians and applications, Rev. Math. Phys. 10 (1997), 1-125.

6. V. M. Buchstaber, V. Z. Enolskit And D. V. Leykin, Rational analogs of Abelian functions, Funct. Analysis Applic. 33 (1999), 83-94.

7. V. M. Buchstaber, D. V. Leykin And V. Z. Enolskit, $\sigma$-function of $(n, s)$-curves, Russ. Math. Surv. 54 (1999), 628-629.

8. D. G. CANTOR, On the analogue of the division polynomials for hyperelliptic curves, $J$. Reine Angew. Math. 447 (1994), 91-145.

9. J. C. Eilbeck, V. Z. Enolskit and E. P. Previato, On a generalized FrobeniusStickelberger addition formula, Lett. Math. Phys. 63 (2003), 5-17.

10. J. FAY, Theta functions on Riemann surfaces, Lecture Notes in Mathematics, vol. 352 (Springer, 1973).

11. F. G. Frobenius and L. Stickelberger, Zur Theorie der elliptischen Functionen, $J$. Reine Angew. Math. 83 (1877), 175-179.

12. D. Grant, A generalization of a formula of Eisenstein, Proc. Lond. Math. Soc. 62 (1991), 121-132.

13. R. C. Gunning, Riemann surfaces and generalized theta functions, Ergebnisse der Mathematik und ihrer Grenzgebiete, vol. 91 (Springer, 1976).

14. L. KIEPERT, Wirkliche Ausführung der ganzzahligen Multiplikation der elliptichen Funktionen, J. Reine Angew. Math. 76 (1873), 21-33.

15. I. G. MaCdonald, Symmetric functions and Hall polynomials (Clarendon Press, Oxford, 1995).

16. S. Matsutani, Elliptic and hyperelliptic solutions of discrete Painlevé I and its extensions to higher order difference equations, Phys. Lett. A 300 (2002), 233-242.

17. D. Mumford, Tata lectures on theta II, Progress in Mathematics, vol. 43 (Birkhäuser, Boston, 1984).

18. Y. ÔNISHI, Complex multiplication formulae for hyperelliptic curves of genus three, Tokyo J. Math. 21 (1998), 381-431 (a list of corrections is available from the author's Web page).

19. Y. ÔNISHI, Determinant expressions for Abelian functions in genus two, Glasgow Math. J. 44 (2002), 353-364.

20. Y. ÔNISHI, Determinant expressions for hyperelliptic functions in genus three, Tokyo $J$. Math. 27 (2004), 299-312. 